\title{
FIBRATIONS AND LOG-SYMPLECTIC STRUCTURES
}

\author{
GIL R. CAVALCANTI AND RALPH L. KLAASSE
}

\begin{abstract}
Log-symplectic structures are Poisson structures $\pi$ on $X^{2 n}$ for which $\bigwedge^{n} \pi$ vanishes transversally. By viewing them as symplectic forms in a Lie algebroid, the $b$-tangent bundle, we use symplectic techniques to obtain existence results for log-symplectic structures on total spaces of fibration-like maps. More precisely, we introduce the notion of a $b$-hyperfibration and show that they give rise to log-symplectic structures. Moreover, we link log-symplectic structures to achiral Lefschetz fibrations and folded-symplectic structures.
\end{abstract}

\section{Contents}

1. Introduction

2. Log-symplectic structures and the $b$-tangent bundle 4

3. Construction of log-symplectic structures 9

4. Achiral Lefschetz fibrations 12

5. b-Hyperfibrations 16

6. Log-symplectic and folded-symplectic structures 20

References 22

\section{INTRODUCTION}

A log-symplectic structure is a Poisson structure $\pi$ on a $2 n$-dimensional manifold $X$ for which $\bigwedge^{n} \pi$ is transverse to the zero section in $\bigwedge^{2 n} T X$. Log-symplectic structures are Poisson structures that are in some sense as close as possible to being symplectic: if $\bigwedge^{n} \pi$ were nowhere vanishing, one could invert $\pi$ to obtain a nondegenerate closed two-form $\omega=\pi^{-1}$, i.e. a symplectic structure on $X$. Transversality is a natural condition to impose on a Poisson structure and hence one is lead to study these objects. From this point of view, log-symplectic structures can be grouped with other symplectic-like structures whose singular behaviour is determined by a transversality condition, such as folded-symplectic and near-symplectic structures. The transversality condition implies that instead of $\pi$ being invertible, it drops rank on - at most - a codimension-one submanifold $Z_{\pi}=\left(\bigwedge^{n} \pi\right)^{-1}(0)$, and there does so generically. The existing literature on log-symplectic structures includes $[9,12,20,25,26,29]$, where they sometimes go under the name of $b$-Poisson or $b$-symplectic structures.

In this paper we take the following viewpoint: out of the singular locus $Z_{\pi}$ one defines a Lie algebroid, the $b$-tangent bundle ${ }^{b} T X$. One then notes that log-symplectic structures with given $Z_{\pi}$ are nothing more than symplectic structures for ${ }^{b} T X$ (see Section 2 for further details). Through this use of $b$-geometry one sees that log-symplectic structures are close enough to being symplectic that one can use symplectic techniques to study them.

The main goal of this paper is to use the language of $b$-geometry to extend results from symplectic geometry relating the existence of fibration-like maps to log-symplectic structures. We first give a brief overview of what has been done in this direction.

Date: 2nd June 2016.

This project was supported by a VIDI grant from NWO, the Dutch science foundation. 
In [31], see also [27], Thurston showed how to equip symplectic fiber bundles with symplectic structures. Gompf [19] then showed that Lefschetz fibrations lead to symplectic structures in dimension four, adapting Thurston's methods. Conversely, Donaldson [10] proved using approximately holomorphic methods that symplectic forms lead to Lefschetz pencils. Further, Gompf $[16,17]$ introduced hyperpencils in all dimensions and showed they admit symplectic structures, aiming to give a topological characterization of symplectic manifolds. Indeed, having established this correspondence, the study of Lefschetz pencils can shed light onto symplectic geometry. One can then branch out to other symplectic-like structures, and connect them to suitably generalized Lefschetz-type fibrations. This has been done for nearsymplectic and folded-symplectic structures.

Near-symplectic geometry. Recall that a near-symplectic structure on a compact oriented four-manifold $X$ is a closed two-form $\omega$ such that there exists a Riemannian metric making $\omega$ harmonic, self-dual, and transverse to the zero section in $\bigwedge^{2,+} T^{*} X$. Such forms vanish on circles, and are symplectic whenever they do not vanish. Near-symplectic structures exist on all compact oriented four-manifolds with $b_{2}^{+}>0$, as one can prove by studying generic metrics $[21,23]$. Auroux-Donaldson-Katzarkov [2] introduced broken Lefschetz pencils to study nearsymplectic structures, and established the same correspondence as between Lefschetz pencils and symplectic structures.

Instead of allowing for broken-type singularities, one can drop the requirement that the charts around Lefschetz singularities are orientation preserving, obtaining so-called achiral Lefschetz singularities. Correspondingly, there is the notion of an achiral Lefschetz fibration and its broken variant. Gay-Kirby [13] proved that all closed oriented four-manifolds admit broken achiral Lefschetz fibrations. Soon thereafter Baykur [4], Lekili [24] and AkbulutKarakurt [1] showed the achirality is unnecessary, i.e. that one can always find broken Lefschetz fibrations over the two-sphere, and broken Lefschetz pencils if in addition $b_{2}^{+}>0$. See also [5] for more on broken Lefschetz fibrations and near-symplectic structures.

Returning to achiral Lefschetz fibrations, Etnyre-Fuller [11] showed that any oriented closed four-manifold admits an achiral Lefschetz fibration over the two-sphere, after performing surgery on a circle. Moreover, they showed that any achiral Lefschetz fibration with a section gives rise to a near-symplectic structure.

Folded-symplectic geometry. Extending the symplectic condition in another direction one obtains folded-symplectic structures. These are closed two-forms $\omega$ on a $2 n$-dimensional manifold $X$ for which $\omega^{n}$ is transverse to the zero section in $\bigwedge^{2 n} T^{*} X$, and such that the restriction of $\omega^{n-1}$ to the zero set $Z_{\omega}$ of $\omega^{n}$ has maximal rank. Here, as for log-symplectic structures, $Z_{\omega}$ is a hypersurface of $X$. Cannas da Silva showed that folded-symplectic structures exist on all compact oriented four-manifolds [7], as their existence is equivalent to having a stable almost-complex structure. This was proven using a version of the $h$-principle for folding maps. In [3], Baykur gave a topological proof for their existence, and showed they arise out of achiral Lefschetz fibrations.

Remark 1.1. In all of the above instances, having some form of homological essentialness of the fibers is required, as the symplectic-like structure will restrict to a symplectic (volume) form on the fibers $F$ of the Lefschetz-type fibration. This is guaranteed for Lefschetz fibrations with fiber genus different from one (as the kernel of the map defines a line bundle $L$, whose Chern class satisfies $\left\langle\left. c_{1}(L)\right|_{F},[F]\right\rangle=\chi(F) \neq 0$ ). Furthermore, it is guaranteed whenever the fibration-like map admits a section. However, in the general case, one must impose this 
condition by hand. There are in some sense two ways of doing this, one of which is by asking that $[F] \neq 0$ and ensuring the fibers are connected. Alternatively, by Poincaré duality, one can demand the existence of a closed two-form which pairs positively with every fiber component. When dealing with pencils this is usually not required, as one can then blow-up the base locus to obtain sections of the resulting fibration.

Log-symplectic geometry. As was mentioned before, we study log-symplectic structures by viewing them as symplectic forms in a Lie algebroid, called the $b$-tangent bundle, and use this viewpoint to apply constructions from symplectic geometry. In doing so we obtain existence results for log-symplectic structures on total spaces of fibration-like maps. We say a pair $\left(X, Z_{X}\right)$ consisting of a manifold and a hypersurface admits a log-symplectic structure $\pi$ if $Z_{\pi}=Z_{X}$, and similarly for folded-symplectic structures. As a warm-up, we prove the $b$-analogue of Thurston's result for fibrations with two-dimensional fibers as Theorem 3.7, which implies the following (see Corollary 3.8).

Theorem. Let $f: X^{2 n} \rightarrow Y^{2 n-2}$ be a fibration between compact connected manifolds. Assume that $Y$ admits a log-symplectic structure $\pi$ and that the generic fiber $F$ is orientable and $[F] \neq 0 \in H_{2}(X ; \mathbb{R})$. Then $\left(X, Z_{X}\right)$ admits a log-symplectic structure, where $Z_{X}=f^{-1}\left(Z_{\pi}\right)$.

Further, after introducing the notion of a $b$-hyperfibration (the $b$-analogue of a hyperpencil with empty base locus [17]) in Section 5, we show the following as Theorem 5.4.

Theorem. Let $f:\left(X, Z_{X}\right) \rightarrow\left(Y, Z_{Y}, \omega_{Y}\right)$ be a b-hyperfibration between compact connected $b$ oriented b-manifolds. Assume that there exists a finite collection $S$ of sections of $f$ interescting all fiber components non-negatively and for each fiber component at least one section in $S$ intersecting positively. Then $\left(X, Z_{X}\right)$ admits a log-symplectic structure.

Our methods also give an alternative proof of a result in [9] that achiral Lefschetz fibrations on compact four-manifolds lead to log-symplectic structures, which we show as Theorem 4.2.

Theorem. Let $f: X^{4} \rightarrow \Sigma^{2}$ be an achiral Lefschetz fibration between compact connected manifolds. Assume that the generic fiber $F$ is orientable and $[F] \neq 0 \in H_{2}(X ; \mathbb{R})$. Then $X$ admits a log-symplectic structure.

It was mentioned before that one can obtain a folded-symplectic structure out of an achiral Lefschetz fibration, but from our point of view this is due to the theorem above and the fact that any log-symplectic structure induces a folded-symplectic structure. This result is known in the Poisson community, and we include its proof here (see Theorem 6.4) for completeness. Further, we show when one can obtain a log-symplectic structure out of a folded-symplectic structure as Theorem 6.5, establishing a converse.

Theorem. Let $X^{2 n}$ be a compact manifold and $Z_{X}$ a hypersurface. Then $\left(X, Z_{X}\right)$ admits a log-symplectic structure $\pi$ if and only if $\left(X, Z_{X}\right)$ admits a folded-symplectic structure $\omega$ and there exists a closed one-form $\theta \in \Omega^{1}\left(Z_{X}\right)$ satisfying $\left.\theta \wedge \omega^{n-1}\right|_{Z_{X}} \neq 0$. Moreover, $\pi^{-1}=\omega$ outside a neighbourhood of $Z_{X}$.

While every oriented four-manifold admits folded-symplectic structures [7], not every compact oriented four-manifold admits a log-symplectic structure, as there are cohomological obstructions to the existence of the latter, see $[9,26]$ or Section 2.

All manifolds in this paper will be compact and without boundary, unless specifically stated otherwise. Note however that they are not necessarily oriented nor orientable. Throughout we will identify de Rham cohomology and singular cohomology with $\mathbb{R}$-coefficients. 
Organization of the paper. In Section 2 we discuss the definition of log-symplectic structures and describe how they can be viewed as symplectic forms for the $b$-tangent bundle. We further discuss the existence of log-symplectic structures on surfaces. In Section 3 we prove Theorem 3.4, which is our main tool for constructing log-symplectic structures, and allows us to prove the $b$-analogue of Thurston's result, Theorem 3.7. In Section 4 we use this tool to prove Theorem 4.2 that four-dimensional achiral Lefschetz fibrations give rise to logsymplectic structures. In Section 5 we define the notion of a $b$-hyperfibration and prove our main theorem, Theorem 5.4, that $b$-hyperfibrations lead to log-symplectic structures. Finally, Section 6 discusses the relation between log-symplectic and folded-symplectic structures.

\section{LOG-SYMPLECTIC STRUCTURES AND THE $b$-TANGENT BUNDLE}

In this section we discuss the notion of a log-symplectic structure and the $b$-geometry language used to study these structures. We defer a more comprehensive account of the interplay of $b$-geometry with log-symplectic structures to [22] and instead only develop what is required for our purposes.

Definition 2.1. A Poisson bivector $\pi$ on a compact manifold $X$ is a section $\pi \in \Gamma\left(\bigwedge^{2} T X\right)$ satisfying $[\pi, \pi]=0$, where $[\cdot, \cdot]$ is the Schouten-Nijenhuis bracket of multivector fields.

Definition 2.2. A log-symplectic structure on a compact $2 n$-dimensional manifold $X$ is a Poisson bivector $\pi$ such that $\bigwedge^{n} \pi$ is transverse to the zero section in $\bigwedge^{2 n} T X$. The set $Z_{\pi}=\left(\bigwedge^{n} \pi\right)^{-1}(0)$ is called the singular locus of $\pi$, and a log-symplectic structure is called bona fide if $Z_{\pi} \neq \emptyset$.

The singular locus of a log-symplectic structure is a codimension-one smooth submanifold, or hypersurface, of $X$. It may be empty and need not be connected. If a given Poisson bivector $\pi$ would be of full rank, we could invert it to obtain a symplectic structure $\omega=\pi^{-1}$ on $X$. One could thus say that log-symplectic structures are "generically generic" Poisson structures, as they drop rank on at most a hypersurface in $X$ and there do so generically. We call $X \backslash Z_{\pi}$ the symplectic locus of $\pi$. We are interested in equipping manifolds with log-symplectic structures and proceed by viewing them as symplectic structures in a specific Lie algebroid. This point of view has also been adopted by $[12,20,25]$ and others.

Let $\left(X, Z_{X}\right)$ be a pair, i.e. a manifold $X$ together with a hypersurface $Z_{X} \subset X$. We say the pair $\left(X, Z_{X}\right)$ admits a log-symplectic structure if there exists a log-symplectic structure $\pi$ on $X$ such that $Z_{\pi}=Z_{X}$. Let $\mathcal{V}_{b}(X) \subset \Gamma(T X)$ be the set of vector fields on $X$ which are tangent to $Z_{X}$. Then $\mathcal{V}_{b}(X)$ defines a locally free sheaf, hence leads to a vector bundle by the Serre-Swan theorem. Furthermore, one notes that $\mathcal{V}_{b}$ forms a Lie subalgebra inside $\Gamma(T X)$.

Definition 2.3. Let $\left(X, Z_{X}\right)$ be a pair. The $b$-tangent bundle ${ }^{b} T X \rightarrow X$ is the vector bundle on $X$ with $\Gamma\left({ }^{b} T X\right)=\mathcal{V}_{b}(X)$. A $b$-manifold is a pair $\left(X, Z_{X}\right)$ equipped with the bundle ${ }^{b} T X$.

We will not distinguish between pairs and $b$-manifolds. The $b$-tangent bundle is an example of a Lie algebroid, i.e. a vector bundle $L \rightarrow X$ together with a map $\rho_{X}: L \rightarrow T X$ called the anchor, such that $\Gamma(L)$ is a Lie algebra, $\rho_{X}$ is a map of Lie algebras when regarded as a map between spaces of sections, and $\rho_{X}$ satisfies the Leibniz rule $[v, f w]=f[v, w]+\left(\rho_{X}(v) f\right) w$ for all $v, w \in \Gamma(L)$ and $f \in C^{\infty}(X)$. In this case the anchor is the natural inclusion, which is an isomorphism away from $Z_{X}$. The $b$-tangent bundle in the case when $X$ is a manifold with boundary and $Z_{X}=\partial X$ has been extensively studied by Melrose and others [28]. 
Definition 2.4. Given two $b$-manifolds $\left(X, Z_{X}\right)$ and $\left(Y, Z_{Y}\right)$, a $b$-map is a map $f: X \rightarrow Y$ such that $f^{-1}\left(Z_{Y}\right)=Z_{X}$ and $f$ is transverse to $Z_{Y}$. We write $f:\left(X, Z_{X}\right) \rightarrow\left(Y, Z_{Y}\right)$.

In other words, for a $b$-map we have $d f_{x}\left(T_{x} X\right)+T_{y} Z_{Y}=T_{y} Y$ for all $y \in Z_{Y}$, where $x \in f^{-1}(y)$. Note that $Z_{X}$ is uniquely determined by $Z_{Y}$ and the requirement that $f$ is a $b$-map. One checks that there is a category with objects being $b$-manifolds and morphisms being $b$-maps between them. Given a $b$-map $f:\left(X, Z_{X}\right) \rightarrow\left(Y, Z_{Y}\right)$, its level sets are either contained in $Z_{X}$ or are disjoint from it. The anchor $\rho_{X}$ gives rise to a well-defined line bundle $\mathbb{L}_{X}:=\operatorname{ker} \rho_{X}$ over $Z_{X}$ which is always trivial. Indeed, $\mathbb{L}_{X}$ is canonically trivialized by a nonvanishing section called the normal b-vector field [20, Proposition 4], locally given by $x \frac{\partial}{\partial x}$ for $x$ a local defining function for $Z_{X}$.

Definition 2.5. A $b$-fibration is a surjective $b$-submersive $b$-map $f:\left(X, Z_{X}\right) \rightarrow\left(Y, Z_{Y}\right)$.

Remark 2.6. It follows immediately from the definition that given a fibration $f: X^{2 n} \rightarrow$ $Y^{2 n-2}$, one can turn it into a $b$-fibration by choosing a hypersurface $Z_{Y} \subset Y$ and considering the $b$-map $f:\left(X, Z_{X}\right) \rightarrow\left(Y, Z_{Y}\right)$, where $Z_{X}=f^{-1}\left(Z_{Y}\right)$.

There are the associated notions of Lie algebroid $k$-forms which we call $b$ - $k$-forms and denote their space of sections by ${ }^{b} \Omega^{k}(X)$, and de Rham differential ${ }^{b} d:{ }^{b} \Omega^{k}(X) \rightarrow{ }^{b} \Omega^{k+1}(X)$ giving the Lie algebroid de Rham cohomology ${ }^{b} H^{k}(X)$. Note that, given a $b$-map $f:\left(X, Z_{X}\right) \rightarrow$ $\left(Y, Z_{Y}\right)$ and a $b$-form $\omega_{Y} \in{ }^{b} \Omega^{k}(Y)$, the pullback $f^{*} \omega_{Y}$ is a well-defined element of ${ }^{b} \Omega^{k}(X)$. We can view forms on $X$ as $b$-forms using pullback via the anchor, $\rho_{X}^{*}: \Omega^{k}(X) \rightarrow{ }^{b} \Omega^{k}(X)$.

Remark 2.7. For the sake of readability, we will often not typographically distinguish between $k$-forms and $b$ - $k$-forms, as well as write merely $d$ instead of ${ }^{b} d$ and say closed instead of $b$-closed. This should not cause any confusion, but note for example that for forms viewed as $b$-forms, the notions of exactness and $b$-exactness do not agree.

A $b$-orientation for $\left(X, Z_{X}\right)$ is an orientation for the bundle ${ }^{b} T X$. A b-almost-complex structure is a vector bundle complex structure for ${ }^{b} T X$, which induces a $b$-orientation. Note that a $b$-manifold $\left(X, Z_{X}\right)$ may be $b$-orientable yet not be orientable, and vice versa.

Definition 2.8. A b-symplectic form is a closed, nondegenerate $b$-two-form $\omega \in{ }^{b} \Omega^{2}(X)$.

As in the symplectic case, a $b$-symplectic form induces a $b$-orientation. We will only consider $b$-orientable $\left(X, Z_{X}\right)$, and $b$-almost-complex structures and $b$-symplectic forms inducing the same $b$-orientation. The reason for introducing the $b$-tangent bundle and $b$-symplectic forms is the following. Viewing a $b$-two-form $\omega \in{ }^{b} \Omega^{2}(X)$ as a map $\omega:{ }^{b} T X \rightarrow{ }^{b} T^{*} X$, if $\omega$ is of maximal rank, this map can be inverted to a map ${ }^{b} \pi:=\omega^{-1}:{ }^{b} T^{*} X \rightarrow{ }^{b} T X$ given by some $b$-bivector ${ }^{b} \pi \in \Gamma\left(\bigwedge^{2} b T X\right)$. Using the anchor one obtains $\rho_{X} \circ^{b} \pi:{ }^{b} T^{*} X \rightarrow T X$, specifying a bivector $\pi_{\omega}:=\rho_{X}\left({ }^{b} \pi\right) \in \Gamma\left(\bigwedge^{2} T X\right)$, which is called the dual bivector to $\omega$. On the other hand, given a log-symplectic structure $\pi$ on $X$, one sees that $\pi: T^{*} X \rightarrow{ }^{b} T X$, and there is a unique $b$-bivector ${ }^{b} \pi:{ }^{b} T^{*} X \rightarrow{ }^{b} T X$ such that $\pi={ }^{b} \pi \circ \rho_{X}^{*}$. The dual $b$-two-form $\omega={ }^{b} \pi^{-1}$ is then a $b$-symplectic form. This is illustrated by the following two diagrams.
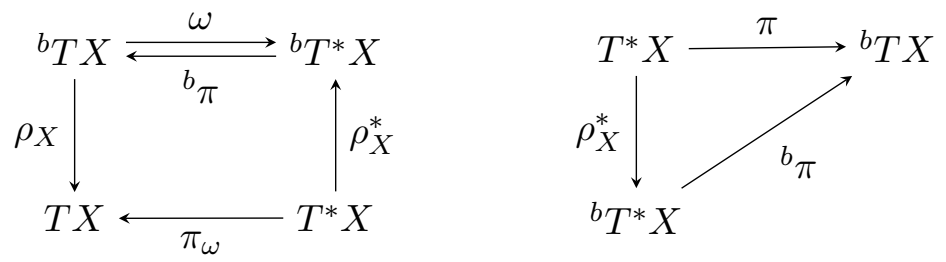
These processes are inverse to each other, and we have the following key result.

Proposition 2.9 ([20, Proposition 20]). Given a manifold X, a b-two-form $\omega$ on the $b$ manifold $\left(X, Z_{X}\right)$ for some hypersurface $Z_{X}$ is b-symplectic if and only if its dual bivector $\pi=\pi_{\omega}$ is a log-symplectic structure with $Z_{\pi}=Z_{X}$. In particular, if $\pi$ is bona fide logsymplectic, then $Z_{X} \neq \emptyset$.

We thus see that the pair $\left(X, Z_{X}\right)$ admits a log-symplectic structure if and only if the $b$-manifold $\left(X, Z_{X}\right)$ admits a $b$-symplectic structure. The $b$-two-form associated to a logsymplectic structure $\pi$ by the above procedure can be viewed as a symplectic form which has a logarithmic singularity at $Z_{X}$. Indeed, the local Darboux model for a $b$-symplectic form $\omega$ on a $2 n$-dimensional manifold $X$ is given by $\omega=d \log x_{1} \wedge d x_{2}+\cdots+d x_{2 n-1} \wedge d x_{2 n}$, using coordinates $x_{i}$ in a neighbourhood $U$ such that $Z_{X} \cap U=\left\{x_{1}=0\right\}$. Its dual bivector is given locally by $\pi=x_{1} \partial_{x_{1}} \wedge \partial_{x_{2}}+\cdots+\partial_{x_{2 n-1}} \wedge \partial_{x_{2 n}}$, where $\partial_{x_{i}}=\partial / \partial x_{i}$.

Remark 2.10. As ${ }^{b} T X$ is isomorphic to $T X$ away from $Z_{X}$ by the anchor, a $b$-oriented $b$-manifold $\left(X, Z_{X}\right)$ obtains an orientation in the usual sense away from $Z_{X}$. Given a $b$ symplectic manifold $\left(X, Z_{X}, \omega\right)$ with dual bivector $\pi$ and the induced $b$-orientation, this orientation on $X \backslash Z_{X}$ can never come from an already existing orientation on $X$ when $Z_{X} \neq \emptyset$. Either $X$ is non-orientable, or if $s \in \Gamma\left(\bigwedge^{2 n} T X\right)$ is a nonvanishing section orienting $X$, we have $\bigwedge^{n} \pi=h s$ for some smooth function $h$ vanishing precisely at $Z_{X}$. From the local description of $\pi$ above one sees that $h$ must change sign so that $Z_{X}$ separates $X$ into two connected components according to the sign of $h$.

The following result extracted from [20] describes the inverse of a log-symplectic structure around its singular locus, see also [9, Theorem 3.2].

Proposition 2.11. Let $\left(X^{2 n}, Z_{X}, \pi\right)$ be a compact log-symplectic manifold. Then around any connected component $Z$ of $Z_{X}$, the two-form $\pi^{-1}$ is equivalent to $d \log |x| \wedge \theta+\sigma$ in a neighbourhood of the zero section of the normal bundle $N Z$ of $Z$, where $|x|$ is the distance to the zero section with respect to a fixed metric on $N Z$. Here $\theta$ and $\sigma$ are closed one- and two-forms on $Z$ satisfying $\theta \wedge \sigma^{n-1} \neq 0$.

The above proposition thus also states that a log-symplectic structure induces a cosymplectic structure on its singular locus. Moreover, let us mention that there are cohomological obstructions for a compact manifold to admit a log-symplectic structure.

Theorem $2.12([26])$. Let $X^{2 n}$ be a compact log-symplectic manifold. Then there exists a class $a \in H^{2}(X ; \mathbb{R})$ such that $a^{n-1} \neq 0$.

Theorem $2.13([9])$. Let $X^{2 n}$ be a compact oriented bona fide log-symplectic manifold. Then there exists a nonzero class $b \in H^{2}(X ; \mathbb{R})$ such that $b^{2}=0$. Moreover, if $n>1$ then $b_{2}(X) \geq 2$.

We now discuss the extent to which there is a difference between $d f$-critical points and ${ }^{b} d f$-critical points, given a $b$-map $f:\left(X, Z_{X}\right) \rightarrow\left(Y, Z_{Y}\right)$.

Proposition 2.14. Let $f:\left(X, Z_{X}\right) \rightarrow\left(Y, Z_{Y}\right)$ be a b-map. Then $\rho_{X, x}: \operatorname{ker}^{b} d f_{x} \rightarrow \operatorname{ker} d f_{x}$ is an isomorphism for all $x \in X$.

Consequently we can unambiguously speak of a critical point of $f$, without specifying whether we mean with respect to ${ }^{b} d f$ or $d f$. The essential ingredients are contained in the following lemma. Two vector spaces $V, V_{1}$ will be called a pair if $V_{1} \subset V$ is a subspace. A linear map $f: V \rightarrow W$ is a map between pairs $\left(V, V_{1}\right)$ and $\left(W, W_{1}\right)$ if $f\left(V_{1}\right) \subset W_{1}$. 
Lemma 2.15. Let $F:\left(V, V_{1}\right) \rightarrow\left(W, W_{1}\right)$ be a linear map between pairs such that under the projection maps $\mathrm{pr}_{V}: V \mapsto V / V_{1}$ and $\mathrm{pr}_{W}: W \mapsto W / W_{1}, F$ descends to an isomorphism $\bar{F}: V / V_{1} \rightarrow W / W_{1}$. Assume that there are vector spaces ${ }^{b} V,{ }^{b} W$ and maps $\rho_{V}:{ }^{b} V \rightarrow V$, $\rho_{W}:{ }^{b} W \rightarrow W,{ }^{b} F:{ }^{b} V \rightarrow{ }^{b} W$ so that $F \circ \rho_{V}=\rho_{W} \circ{ }^{b} F$. Assume that im $\rho_{V}=V_{1}$, $\operatorname{im} \rho_{W}=W_{1}$ and ${ }^{b} F: \operatorname{ker} \rho_{V} \rightarrow \operatorname{ker} \rho_{W}$ is an isomorphism. Then $\rho_{V}: \operatorname{ker}^{b} F \rightarrow \operatorname{ker} F$ is an isomorphism.

The situation is summarized by the following diagram, in which the rows are exact. The two vertical maps on the far left and right are assumed to be isomorphisms, while the conclusion of the lemma is that the top horizontal map is an isomorphism.

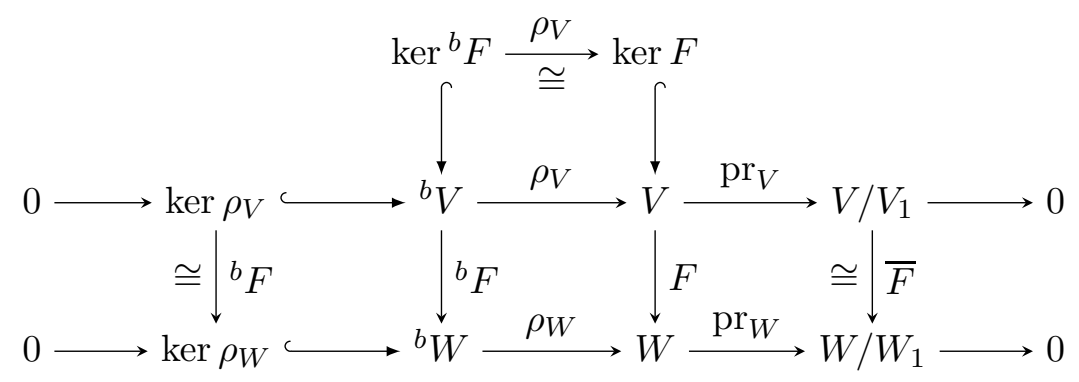

Proof. If ${ }^{b} v \in \operatorname{ker}^{b} F$, then $F \rho_{V}\left({ }^{b} v\right)=\rho_{W}{ }^{b} F\left({ }^{b} v\right)=0$, so that $\rho_{V}: \operatorname{ker}^{b} F \rightarrow \operatorname{ker} F$. Given $v \in$ ker $F$ we have $\operatorname{pr}_{W} F(v)=\operatorname{pr}_{W}(0)=0$, so by assumption $\operatorname{pr}_{V}(v)=0$, hence $v \in V_{1}$. As $v \in V_{1}$, there exists a ${ }^{b} v_{0} \in{ }^{b} V$ such that $\rho_{V}\left({ }^{b} v_{0}\right)=v$, and $\rho_{V}^{-1}(v)={ }^{b} v_{0}+\operatorname{ker} \rho_{V}$. Consider a vector ${ }^{b} v={ }^{b} v_{0}+k$ for $k \in \operatorname{ker} \rho_{V}$, so that $\rho_{V}\left({ }^{b} v\right)=v$. Then $\rho_{W}{ }^{b} F\left({ }^{b} v\right)=F \rho_{V}\left({ }^{b} v\right)=$ $F(v)=0$, so that ${ }^{b} F\left({ }^{b} v\right) \in \operatorname{ker} \rho_{W}$. But then there exists a unique $k \in \operatorname{ker} \rho_{V}$ such that ${ }^{b} F(k)=-{ }^{b} F\left({ }^{b} v_{0}\right)$, for which ${ }^{b} F\left({ }^{b} v\right)=0$. Hence there exists a unique ${ }^{b} v \in \operatorname{ker}{ }^{b} F \cap \rho_{V}^{-1}(v)$. We conclude that $\rho_{V}: \operatorname{ker}^{b} F \rightarrow \operatorname{ker} F$ is an isomorphism.

Proof of Proposition 2.14. Let $x \in X$ be given and denote $y=f(x)$. If $x \in X \backslash Z_{X}$ the statement follows as $\rho_{X}$ is an isomorphism away from $Z_{X}$. If $x \in Z_{X}$, we wish to apply Lemma 2.15 to the situation where $V=T_{x} X, V_{1}=T_{x} Z_{X}, W=T_{y} Y, W_{1}=T_{y} Z_{Y},{ }^{b} V=$ ${ }^{b} T_{x} X,{ }^{b} W={ }^{b} T_{y} Y, F=d f_{x},{ }^{b} F={ }^{b} d f_{x}, \rho_{V}=\rho_{X, x}$ and $\rho_{W}=\rho_{Y, y}$. As $f$ is a $b$-map, we have $f^{-1}\left(Z_{Y}\right)=Z_{X}$ so that $d f_{x}\left(T_{x} Z_{X}\right) \subset T_{y} Z_{Y}$ making $F$ a map between pairs. Splitting $T_{x} X=N_{x} Z_{X} \oplus T_{x} Z_{X}$ pointwise we see that $T_{x} X / T_{x} Z_{X} \cong N_{x} Z_{X}$ and $d f_{x}\left(T_{x} X\right)+T_{y} Z_{Y}=$ $d f_{x}\left(N_{x} Z_{X}\right)+T_{y} Z_{Y}$. Because $f$ is a $b$-map we have $d f_{x}\left(T_{x} X\right)+T_{y} Z_{Y}=T_{y} Y$. Similarly splitting $T_{y} Y=N_{y} Z_{Y} \oplus T_{y} Z_{Y}$ we conclude that $d f_{x}\left(N_{x} Z_{X}\right)+T_{y} Z_{Y}=N_{y} Z_{Y} \oplus T_{y} Z_{Y}$ and we see that $d f_{x}$ restricts to an isomorphism from $N_{x} Z_{X} \cong V / V_{1}$ to $N_{y} Z_{Y} \cong W / W_{1}$. By construction $d f \circ \rho_{X}=\rho_{Y} \circ{ }^{b} d f$, hence also pointwise at $x$ and $y$, and furthermore by definition of the $b$-tangent bundles we have $\operatorname{im} \rho_{X, x}=T_{x} Z_{X}$ and $\operatorname{im} \rho_{Y, y}=T_{y} Z_{Y}$. Note that $f^{*} \mathbb{L}_{Y} \cong \mathbb{L}_{X}$, because local defining functions for $Z_{Y}$ pull back to local defining functions for $Z_{X}$. Hence ${ }^{b} d f_{x}: \mathbb{L}_{X, x} \rightarrow \mathbb{L}_{Y, y}$ is an isomorphism. Finally, $\operatorname{ker} \rho_{X, x}=\mathbb{L}_{X, x}$ and $\operatorname{ker} \rho_{Y, y}=\mathbb{L}_{Y, y}$, so that ${ }^{b} d f_{x}: \operatorname{ker} \rho_{X, x} \rightarrow \operatorname{ker} \rho_{Y, y}$ is an isomorphism. By Lemma 2.15 we conclude that $\rho_{X, x}$ : $\operatorname{ker}^{b} d f_{x} \rightarrow \operatorname{ker} d f_{x}$ is an isomorphism.

The situation is summarized by the following diagram with exact rows. 


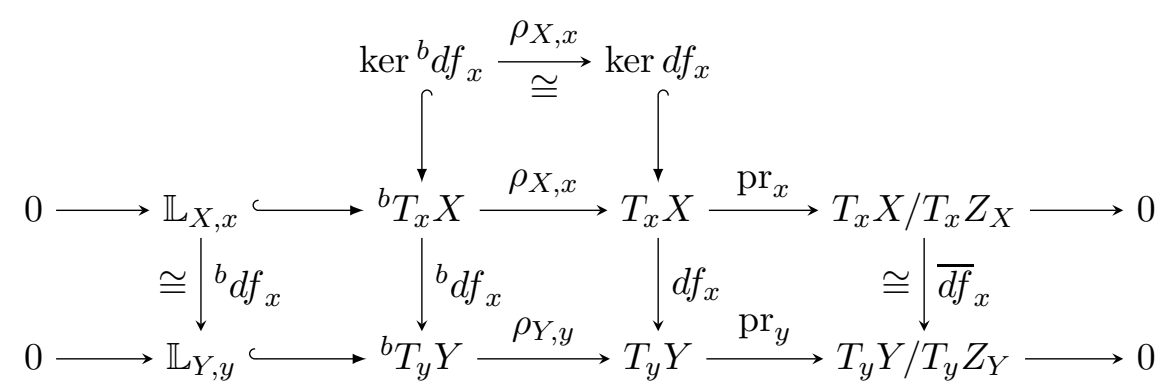

Remark 2.16. The statement that $d f_{x}: N_{x} Z_{X} \rightarrow N_{y} Z_{Y}$ is an isomorphism for $y=f(x) \in$ $Z_{Y}$ can be colloquially phrased as follows. As $f$ is transverse to $Z_{Y}$, the normal direction to $Z_{Y}$ at points in $Z_{Y}$ must be contained in the image of $T X$ under $d f$. The fact that $f^{-1}\left(Z_{Y}\right)=Z_{X}$ then implies that it must in fact be obtained from the normal direction to $Z_{X}$. As both $N_{x} Z_{X}$ and $N_{y} Z_{Y}$ are one-dimensional subspaces and $d f_{x}$ gives a surjection, it is an isomorphism.

We next discuss sections of $b$-maps, in preparation of Section 5.

Proposition 2.17. Let $f:\left(X, Z_{X}\right) \rightarrow\left(Y, Z_{Y}\right)$ be a b-map, and let $s$ be a section of $f$. Then $s:\left(Y, Z_{Y}\right) \rightarrow\left(X, Z_{X}\right)$ is a b-map, and $\operatorname{ker}^{b} d f_{x} \oplus^{b} d s_{y}\left({ }^{b} T_{y} Y\right)={ }^{b} T_{x} X$ for all $x \in X$, where $y=f(x)$.

Proof. By definition $f \circ s=\operatorname{id}_{Y}$, hence $s^{-1}\left(Z_{X}\right)=s^{-1}\left(f^{-1}\left(Z_{Y}\right)\right)=(f \circ s)^{-1}\left(Z_{Y}\right)=Z_{Y}$. Note that given finite-dimensional vector spaces $U, W, V^{\prime}, V$ with $V^{\prime} \subset V$ so that $U \hookrightarrow V^{\prime} \rightarrow W$ and $U \hookrightarrow V \rightarrow W$ we have $V^{\prime}=V$ by counting dimensions. Let $y \in Z_{Y}$ and $x \in f^{-1}(y)$ be given. By definition ker $d f_{x} \hookrightarrow T_{x} X \rightarrow T_{y} Y$ using $d f_{x}$. There is a clear surjection of $d s_{y}\left(T_{y} Y\right)$ onto $T_{y} Y$. As $d s_{y}\left(T_{y} Y\right)+T_{x} Z_{X} \subset T_{x} X$ and ker $d f_{x} \hookrightarrow T_{x} Z_{X}$ because $f^{-1}\left(Z_{Y}\right)=Z_{X}$, we see that ker $d f_{x} \hookrightarrow d s_{y}\left(T_{y} Y\right)+T_{x} Z_{X} \rightarrow T_{y} Y$, so that $d s_{y}\left(T_{y} Y\right)+T_{x} Z_{X}=T_{x} X$. We conclude that $s$ is a $b$-map. Similarly, consider $x \in X$ and denote $y=f(x)$. As $f$ is a $b$-map we have $\operatorname{ker}^{b} d f_{x} \hookrightarrow{ }^{b} T_{x} X \rightarrow{ }^{b} T_{y} Y$ using ${ }^{b} d f_{x}$. Again there is a surjection of ${ }^{b} d s_{y}\left({ }^{b} T_{y} Y\right)$ onto ${ }^{b} T_{y} Y$. Note that $\operatorname{ker}^{b} d f_{x} \oplus^{b} d s_{y}\left({ }^{b} T_{y} Y\right) \subset{ }^{b} T_{x} X$ and furthermore $\operatorname{ker}^{b} d f_{x} \hookrightarrow \operatorname{ker}^{b} d f_{x} \oplus^{b} d s_{y}{ }^{b} T_{y} Y \rightarrow$ ${ }^{b} T_{y} Y$, so that $\operatorname{ker}^{b} d f_{x} \oplus^{b} d s_{y}^{b} T_{y} Y={ }^{b} T_{x} X$.

Let $f:\left(X^{4}, Z_{X}\right) \rightarrow\left(\Sigma^{2}, Z_{\Sigma}\right)$ be a $b$-map for which ker $^{b} d f$ is even-dimensional. For example, this situation arises when there is a $b$-symplectic form $\omega_{\Sigma}$ on $\left(\Sigma, Z_{\Sigma}\right)$ and a $b$ almost-complex structure $J$ on $\left(X, Z_{X}\right)$ so that $J$ is $\left(\omega_{\Sigma}, f\right)$-tame. Because $f$ is transverse to $Z_{\Sigma}$, we conclude that $Z_{\Sigma}$ cannot contain critical values of $f$. Namely, if $x$ were a critical point of $f$, by a dimension count and Proposition 2.14 we would have ker $d f_{x}=T_{x} X$, hence $d f_{x}\left(T_{x} X\right)+T_{f(x)} Z_{Y}=T_{f(x)} Z_{Y}$, not $T_{f(x)} Y$. Said differently, such a map $f: X^{4} \rightarrow \Sigma^{2}$ can only be turned into a $b$-map if $Z_{\Sigma}$ is disjoint from the set of critical values of $f$.

2.1. Log-symplectic structures on surfaces. In this section we discuss log-symplectic structures on a compact surface $\Sigma$. These were first studied in [29], but we are interested in existence, i.e. which pairs $\left(\Sigma, Z_{\Sigma}\right)$ admit log-symplectic structures. Any surface $\Sigma$, compact or not, orientable or not, admits a log-symplectic structure. This is because any vector bundle admits transverse sections, hence so does $\bigwedge^{2} T \Sigma$; the Poisson condition is immediate in dimension two. However, not every pair $\left(\Sigma, Z_{\Sigma}\right)$ admits a log-symplectic structure, with an easy counterexample being $\left(\mathbb{R} P^{2}, \emptyset\right)$. The hypersurface $Z_{\Sigma}$ must be the zero set $N(s)$ of 
some section $s \in \Gamma\left(\bigwedge^{2} T \Sigma\right)$, so that $Z_{\Sigma}$ represents $w_{1}\left(\bigwedge^{2} T \Sigma\right)$ and $\Sigma \backslash Z_{\Sigma}$ is oriented by $s$. More precisely, we have the following.

Proposition 2.18. Let $\left(\Sigma^{2}, Z_{\Sigma}\right)$ be given. Then $\left(\Sigma, Z_{\Sigma}\right)$ admits a log-symplectic structure if and only if $w_{1}(\Sigma)=\mathrm{PD}\left[Z_{\Sigma}\right]$, the Poincaré dual using $\mathbb{Z}_{2}$-coefficients.

Proof. The necessity of the condition follows after noting that $w_{1}\left(\bigwedge^{2} T \Sigma\right)=w_{1}(T \Sigma)=w_{1}(\Sigma)$ and the log-symplectic structure provides a transverse section of $\Lambda^{2} T \Sigma$ with zero set $Z_{\Sigma}$. To see sufficiency, then by hypothesis there exists a transverse section $\pi \in \Gamma\left(\bigwedge^{2} T \Sigma\right)$ whose zero set $N(\pi)$ is equal to $Z_{\Sigma}$. As we are in dimension two, the Poisson condition $[\pi, \pi]=0$ is immediate. Thus $\pi$ is a $\log$-symplectic structure with $Z_{\pi}=Z_{\Sigma}$.

If $\Sigma$ is orientable then $w_{1}(\Sigma)=0$, making the condition that $Z_{\Sigma}$ must be $\mathbb{Z}_{2}$-nullhomologous. Remark further that $\left(\Sigma, Z_{\Sigma}\right)$ carries a log-symplectic structure iff it is $b$-orientable.

\section{Construction of LOG-Symplectic structures}

In this section we discuss extensions of symplectic results to the world of $b$-geometry to obtain existence results for log-symplectic structures. Let $X$ and $Y$ be compact connected manifolds, and assume that $Y$ is equipped with a log-symplectic structure. Using Proposition 2.9 we can view $Y$ as being a $b$-manifold with a $b$-symplectic structure, obtaining a triple $\left(Y, Z_{Y}, \omega_{Y}\right)$. Given a map $f: X \rightarrow Y$ such that $f$ is transverse to $Z_{Y}$, we can turn it into a $b$-map $f:\left(X, Z_{X}\right) \rightarrow\left(Y, Z_{Y}\right)$ by defining $Z_{X}:=f^{-1}\left(Z_{Y}\right)$. We wish to equip $X$ with a log-symplectic structure by constructing a $b$-symplectic form coming from $f$ and $\omega_{Y}$ and then use Proposition 2.9. In analogy with [17], we will do this using $b$-almost-complex structures to guide the process.

Definition 3.1. Let $T: V \rightarrow W$ be a linear map between two finite-dimensional real vector spaces, and let $\omega$ be a skew-symmetric bilinear form on $W$. Then a linear complex structure $J$ on $V$ is called $(\omega, T)$-tame if $T^{*} \omega(v, J v)>0$ for all $v \in V \backslash \operatorname{ker} T$. For $f:\left(X, Z_{X}\right) \rightarrow\left(Y, Z_{Y}\right)$ a $b$-map and $\omega_{Y} \in{ }^{b} \Omega^{2}(Y)$, a $b$-almost-complex structure $J$ on $X$ is called $\left(\omega_{Y}, f\right)$-tame if it is $\left(\omega_{Y},{ }^{b} d f_{x}\right)$-tame for all $x \in X$.

When $T=\operatorname{id}_{V}$ or $f=\operatorname{id}_{X}$ and $Z_{X}=\emptyset$, we recover the usual notion of $J$ being $\omega$-tame. Note that any $\omega \in{ }^{b} \Omega^{2}(X)$ taming a $b$-almost-complex structure $J$ is necessarily non-degenerate. Hence if $\omega \in{ }^{b} \Omega^{2}(X)$ is a closed $b$-two-form taming some $J$, then $\omega$ is $b$-symplectic and $J$ induces the same $b$-orientation as $\omega$. Note moreover that if $J$ is $\left(\omega_{Y}, f\right)$-tame, then $\operatorname{ker}^{b} d f$ is a $J$-complex subspace so that preimages of regular values of $f$ are $J$-holomorphic submanifolds. Indeed, if $v \in \operatorname{ker}^{b} d f$ and $J v \notin \operatorname{ker}^{b} d f$, we would have $0=f^{*} \omega(v, J v)=f^{*} \omega(J v, J(J v))>0$, which is a contradiction. If $\omega$ is $b$-symplectic there exist tamed $J$ for $\omega$, and the space of such $J$ is convex and hence contractible [27].

Remark 3.2. An important property of tameness is that the taming condition is open, i.e. it is preserved under sufficiently small perturbations of $\omega$ and $J$, and of varying the point in $X$. Namely, the taming condition $\omega(v, J v)>0$ for the pair $(\omega, J)$ holds provided it holds for all $v \in{ }^{b} \Sigma X \subset{ }^{b} T X$, the unit sphere bundle with respect to some preassigned metric, as $X$ is compact. As ${ }^{b} \Sigma X$ is also compact, the continuous map $\widetilde{\omega}:{ }^{b} \Sigma X \rightarrow \mathbb{R}$ given by $\widetilde{\omega}(v):=\omega(v, J v)$ for $v \in{ }^{b} \Sigma X$ is bounded from below by a positive constant on ${ }^{b} \Sigma X$. But then this map will remain positive under small perturbations of $\omega$ and $J$. Similarly the condition of $\omega$ taming $J$ on $\operatorname{ker}^{b} d f$ is open. Consider $x \in X$ so that $\omega(v, J v)>0$ for all 
$v \in \operatorname{ker}^{b} d f_{x}$. As $\widetilde{\omega}$ is continuous and ${ }^{b} \Sigma X$ is compact, there exists a neighbourhood $U$ of $\operatorname{ker}^{b} d f_{x} \cap{ }^{b} \Sigma X$ in ${ }^{b} \sum X$ on which $\widetilde{\omega}$ is positive. Points $x^{\prime} \in X$ close to $x$ will then have $\operatorname{ker}^{b} d f_{x^{\prime}} \subset U$ because $\operatorname{ker}^{b} d f$ is closed.

We will not use the associated notion of compatibility, where $J$ also leaves $\omega$ invariant, as we use $b$-almost-complex structures as auxiliary structures to show non-degeneracy, and make use of the openness of this condition. For this reason, all $b$-almost-complex structures will only be required to be continuous as this avoids arguments to ensure smoothness. Using a $b$-almost-complex structure on $X$, one can demand the existence of suitable forms on both the base and the fibers, to be combined into a $b$-symplectic form on the total space $X$. Note that the fibers here do not have to be two-dimensional.

Remark 3.3. Products of log-symplectic manifolds are not log-symplectic in general, as the product form is not log-symplectic when both are bona fide. However, the product of a log-symplectic manifold with a symplectic manifold is always log-symplectic.

Given a map $f: X \rightarrow Y$ between compact connected manifolds, we will use the following notation.

- $F_{y}=f^{-1}(y)$ for $y \in Y$ is the level set, or fiber, of $f$ over $y$;

- $[F]$ is the homology class of a generic fiber, i.e. the inverse image of a regular value.

The latter will only be used in instances where it is well defined and independent of the regular value. We now formulate the $b$-version of [17, Theorem 3.1]. Note that there are no assumptions on the dimension of the manifolds involved, other than that they must be even.

Theorem 3.4. Let $f:\left(X, Z_{X}\right) \rightarrow\left(Y, Z_{Y}\right)$ be a b-map between compact connected b-oriented $b$-manifolds, $J$ a b-almost-complex structure on $\left(X, Z_{X}\right), \omega_{Y}$ a b-symplectic form on $\left(Y, Z_{Y}\right)$, and $c \in H^{2}(X ; \mathbb{R})$. Assume that

i) $J$ is $\left(\omega_{Y}, f\right)$-tame;

ii) for each $y \in Y, F_{y}$ has a neighbourhood $W_{y}$ with a closed two-form $\eta_{y} \in \Omega^{2}\left(W_{y}\right)$ such that $\left[\eta_{y}\right]=\left.c\right|_{W_{y}} \in H^{2}\left(W_{y} ; \mathbb{R}\right)$, and $\rho_{X}^{*} \eta_{y}$ tames $\left.J\right|_{\operatorname{ker}^{b} d f_{x}}$ for all $x \in W_{y}$.

Then $\left(X, Z_{X}\right)$ admits a log-symplectic structure.

Remark 3.5. As one can see by inspecting the proof, the second condition in the above theorem can be replaced by the existence of a closed two-form $\eta \in \Omega^{2}(X)$ such that $\rho_{X}^{*} \eta$ tames $\left.J\right|_{\operatorname{ker}^{b} d f_{x}}$ for all $x \in X$. Such forms are called symplectic on the fibers of $f$. Comparing Theorem 3.4 to [17, Theorem 3.1] one sees that the form on the base $Y$ is $b$-symplectic, while the fiberwise form $\eta$ is still symplectic in the usual sense.

The proof of this result is modelled on that by Gompf of [17, Theorem 3.1].

Proof. Using Proposition 2.9 it suffices to construct a $b$-symplectic form on the $b$-manifold $\left(X, Z_{X}\right)$. Let $\xi \in \Omega_{\mathrm{cl}}^{2}(X)$ be such that $[\xi]=c$. Then for each $y \in Y$ we have $\left[\eta_{y}\right]=\left.c\right|_{W_{y}}=$ $\left.[\xi]\right|_{W_{y}}$, so on $W_{y}$ we have $\eta_{y}=\xi+d \alpha_{y}$ for some $\alpha_{y} \in \Omega^{1}\left(W_{y}\right)$. As each $X \backslash W_{y}$ and hence $f\left(X \backslash W_{y}\right)$ is compact, each $y \in Y$ has a neighbourhood disjoint from $f\left(X \backslash W_{y}\right)$. Cover $Y$ by a finite amount of such open sets $U_{i}$ so that each $f^{-1}\left(U_{i}\right)$ is contained in some $W_{y_{i}}$. Let $\left\{\varphi_{i}\right\}$ be a partition of unity subordinate to the cover $\left\{U_{i}\right\}$ of $Y$, so that $\left\{\varphi_{i} \circ f\right\}$ is a partition of unity of $X$. Define a two-form $\eta \in \Omega^{2}(X)$ on $X$ via

$$
\eta:=\xi+d\left(\sum_{i}\left(\varphi_{i} \circ f\right) \alpha_{y_{i}}\right)=\xi+\sum_{i}\left(\varphi_{i} \circ f\right) d \alpha_{y_{i}}+\sum_{i}\left(d \varphi_{i} \circ d f\right) \wedge \alpha_{y_{i}} .
$$


Then $d \eta=0$ and $[\eta]=c$. The last of the above three terms vanishes when applied to a pair of vectors in ker $d f_{x}$ for any $x \in X$, so on each ker $d f_{x}$ we have

$$
\eta=\xi+\sum_{i}\left(\varphi_{i} \circ f\right) d \alpha_{y_{i}}=\sum_{i}\left(\varphi_{i} \circ f\right)\left(\xi+d \alpha_{y_{i}}\right)=\sum_{i}\left(\varphi_{i} \circ f\right) \eta_{y_{i}} .
$$

Consider ${ }^{b} \eta:=\rho_{X}^{*} \eta$, a closed $b$-two-form on $\left(X, Z_{X}\right)$. By Proposition 2.14 and the above we see that on $\operatorname{ker}^{b} d f$, the $b$-form ${ }^{b} \eta$ is a convex combination of $J$-taming $b$-forms, so $\left.J\right|_{\text {ker } b d f}$ is ${ }^{b} \eta$-tame. Define for $t>0$ a global closed $b$-two-form on $X$ by $\omega_{t}:=f^{*} \omega_{Y}+t^{b} \eta$. We show that $\omega_{t}$ is $b$-symplectic by showing $\omega_{t}$ tames $J$ for $t$ small enough. By Remark 3.2 it is enough to show that there exists a $t_{0}>0$ so that $\omega_{t}(v, J v)>0$ for every $t \in\left(0, t_{0}\right)$ and $v$ in the unit sphere bundle ${ }^{b} \Sigma X \subset{ }^{b} T X$ with respect to some metric. Note that for $v \in{ }^{b} T X$ we have $\omega_{t}(v, J v)=f^{*} \omega_{Y}(v, J v)+t^{b} \eta(v, J v)$. As $J$ is $\left(\omega_{Y}, f\right)$-tame, the first term is positive for $v \in{ }^{b} T X \backslash \operatorname{ker}^{b} d f$ and is zero otherwise. The second term ${ }^{b} \eta(v, J v)$ is positive on ker ${ }^{b} d f$ because $\left.J\right|_{\text {ker }^{b} d f}$ is ${ }^{b} \eta$-tame, hence is also positive for all $v$ in some neighbourhood $U$ of $\operatorname{ker}^{b} d f \cap^{b} \Sigma X$ in ${ }^{b} \Sigma X$ by openness of the taming condition. We conclude that $\omega_{t}(v, J v)>0$ for all $t>0$ when $v \in U$. The function ${ }^{b} \eta(v, J v)$ is bounded on the compact set ${ }^{b} \Sigma X \backslash U$. Furthermore, $f^{*} \omega_{Y}(v, J v)$ is bounded from below there by a positive constant, as it is positive away from $\operatorname{ker}^{b} d f$, and thus also away from $\operatorname{ker}^{b} d f \cap^{b} \Sigma X \subset U$. But then $\omega_{t}(v, J v)>0$ for all $0<t<t_{0}$ for $t_{0}$ sufficiently small, so that $\omega_{t}$ is indeed $b$-symplectic for $t$ small enough.

Remark 3.6. Given a $b$-map $f:\left(X, Z_{X}\right) \rightarrow\left(Y, Z_{Y}\right)$, the singular locus of $X$ is given by $Z_{X}=f^{-1}\left(Z_{Y}\right)$, so that it consists purely of fibers. This means the fibers of $f$, which are natural candidates for being $b$-symplectic submanifolds of $X$, never hit $Z_{X}$.

With Theorem 3.4 in hand we can prove the $b$-version of Thurston's result for symplectic fiber bundles with two-dimensional fibers [31], adapting the proof by Gompf in [15].

Theorem 3.7. Let $f:\left(X^{2 n}, Z_{X}\right) \rightarrow\left(Y^{2 n-2}, Z_{Y}\right)$ be a b-fibration between compact connected $b$-manifolds. Assume that $\left(Y, Z_{Y}\right)$ is b-symplectic and that the generic fiber $F$ is orientable and $[F] \neq 0 \in H_{2}(X ; \mathbb{R})$. Then $\left(X, Z_{X}\right)$ admits a log-symplectic structure.

This theorem has the following immediate corollary, phrased without using the $b$-language.

Corollary 3.8. Let $f: X^{2 n} \rightarrow Y^{2 n-2}$ be a fibration between compact connected manifolds. Assume that $Y$ admits a log-symplectic structure $\pi$ and that the generic fiber $F$ is orientable and $[F] \neq 0 \in H_{2}(X ; \mathbb{R})$. Then $\left(X, Z_{X}\right)$ admits a log-symplectic structure for $Z_{X}=f^{-1}\left(Z_{\pi}\right)$.

Proof. Let $Z_{Y}:=Z_{\pi}$. Then by Proposition 2.9, $\left(Y, Z_{Y}\right)$ is $b$-symplectic. By Remark 2.6, the map $f$ can be seen as a $b$-fibration $f:\left(X, Z_{X}\right) \rightarrow\left(Y, Z_{Y}\right)$, where $Z_{X}=f^{-1}\left(Z_{Y}\right)$. Using Theorem 3.7 we conclude that $\left(X, Z_{X}\right)$ admits a log-symplectic structure.

For example, any homologically essential oriented surface bundle over a surface is logsymplectic (regardless of whether the base is orientable). The fact that any such base surface is log-symplectic is discussed in Section 2.1.

Proof of Theorem 3.7. We check the conditions of Theorem 3.4. If necessary, pass to a finite cover of $Y$ so that the fibers of $f$ are connected. Let $\omega_{Y}$ be a $b$-symplectic form on $Y$ and choose an $\omega_{Y}$-taming $b$-almost-complex structure $J_{Y}$ on $Y$. Fix an orientation for the generic fiber $F$, which orients ker $d f$ and $\operatorname{ker}^{b} d f$, using Proposition 2.14. As $[F] \neq 0$, this forces $f:\left(X, Z_{X}\right) \rightarrow\left(Y, Z_{Y}\right)$ to be an orientable $b$-fibration. In particular, $f$ provides a $b$-orientation for $\left(X, Z_{X}\right)$ by using the fiber-first convention. Let $g$ be a metric on ${ }^{b} T X$ and 
let $H \subset{ }^{b} T X$ be the subbundle of orthogonal complements to $\operatorname{ker}^{b} d f$. Note here that $\operatorname{ker}^{b} d f$ is the tangent space to the fibers of $f$, because $f$ is a $b$-fibration. Then $\left.{ }^{b} d f\right|_{H}: H \rightarrow{ }^{b} T Y$ is an isomorphism on each fiber. Define a $b$-almost-complex structure $J$ on $X$ as follows. On $H$, define $\left.J\right|_{H}=f^{*} J_{Y}$ using the isomorphism given by ${ }^{b} d f$. On the two-dimensional tangent spaces to the fibers, use the metric and define $J$ by $\frac{\pi}{2}$-counterclockwise rotation, demanding that $f$ is orientation preserving via the fiber-first convention. This determines $J$ uniquely on ${ }^{b} T X$ by linearity. Moreover, $J$ is $\left(\omega_{Y}, f\right)$-tame as $f^{*} \omega_{Y}(v, J v)=\omega_{Y}\left({ }^{b} d f v, J_{Y}{ }^{b} d f v\right)>0$, for all $v \in{ }^{b} T X \backslash \operatorname{ker}^{b} d f \cong H$.

We construct the required neighbourhoods $W_{y}$ and forms $\eta_{y}$ for each fiber. Let $c \in$ $H^{2}(X ; \mathbb{R})$ be through duality a class such that $\langle[F], c\rangle=1$, using that $[F] \neq 0$. Given $y \in Y$, let $D_{y} \subset Y$ be an open disk containing $y$, fully contained in a trivializing neighbourhood of $f$ around $y$. Define $W_{y}:=f^{-1}\left(D_{y}\right) \cong D_{y} \times F_{y}$. Using that $F_{y}$ is two-dimensional, choose an area form on $F_{y}$ inducing the preimage orientation of the fiber, and let $\eta_{y} \in \Omega^{2}\left(W_{y}\right)$ be the pullback of this form via the projection $p: W_{y} \rightarrow F_{y}$. Because $\left\langle\left[F_{y}\right], \eta_{y}\right\rangle=1=\left\langle\left[F_{y}\right], c\right\rangle$ and $H_{2}\left(W_{y} ; \mathbb{R}\right)$ is generated by $\left[F_{y}\right]$, it follows that $\left[\eta_{y}\right]=\left.c\right|_{W_{y}} \in H^{2}\left(W_{y} ; \mathbb{R}\right)$. To check that $\rho_{X}^{*} \eta_{y}$ tames $J$ on $\operatorname{ker}^{b} d f_{x}$ for $x \in W_{y}$, recall that there $J$ is defined via rotation. As $\rho_{X}^{*} \eta_{y}$ is the pullback of the area form of a fiber, taming follows as its restriction to a fiber is an area form for that fiber. Applying Theorem 3.4 we obtain a log-symplectic structure on $\left(X, Z_{X}\right)$.

Remark 3.9. Note how the two-dimensionality of the fibers is used to be able to define an almost-complex structure there, and for choosing an area form.

\section{Achiral Lefschetz fibrations}

In this section we revisit the result proven in [9] that any homologically essential achiral Lefschetz fibration with orientable fibers gives rise to a log-symplectic structure in dimension four, which can be chosen to be bona fide. Our proof using b-geometry is a direct adaptation of the proof that four-dimensional Lefschetz fibrations give rise to symplectic structures, phrased using almost-complex structures.

Definition 4.1. An achiral Lefschetz fibration is a map $f: X^{2 n} \rightarrow \Sigma^{2}$ between compact connected manifolds so that for each critical point $x \in X$ there exist complex coordinate charts centered at $x$ and $f(x)$ in which $f$ takes the form $f: \mathbb{C}^{n} \rightarrow \mathbb{C}, f\left(z_{1}, \ldots, z_{n}\right)=z_{1}^{2}+\cdots+z_{n}^{2}$.

Note that we do not require $X$ nor $\Sigma$ to be orientable. If they are however, after choosing orientations one can assign a sign to each critical point of $f$. A given critical point $x \in X$ obtains a sign by demanding that the complex structure of the chart on $\Sigma$ is compatible with its orientation; we then say $x$ is positive if the complex structure on $X$ is compatible with its orientation, and negative otherwise. Note that any Lefschetz fibration is also an achiral Lefschetz fibration.

Theorem 4.2 ([9, Theorem 6.7]). Let $f: X^{4} \rightarrow \Sigma^{2}$ be an achiral Lefschetz fibration between compact connected manifolds. Assume that the generic fiber $F$ is orientable and $[F] \neq 0 \in$ $H_{2}(X ; \mathbb{R})$. Then $X$ admits a log-symplectic structure.

This theorem should be viewed as a direct analogue of [19, Theorem 10.2.18] that homologically essential four-dimensional Lefschetz fibrations provide symplectic structures. We prove it by first showing that an achiral Lefschetz fibration gives rise to what we call a $b$-Lefschetz fibration. Noting Proposition 2.14 we use the following notation, given a map $f: X \rightarrow Y$ between manifolds (in what follows, $Y=\Sigma$ ). 
- $\Delta=\operatorname{Crit}(f) \subset X$ is the set of critical points of $f$;

- $\Delta_{y}=\Delta \cap F_{y}$ for $y \in Y$ is the set of critical points of $f$ lying on the fiber $F_{y}$;

- $\Delta^{\prime}=\operatorname{Sing}(f) \subset Y$ is the set of singular values of $f$.

Definition 4.3. A $b$-Lefschetz fibration is a b-map $f:\left(X^{2 n}, Z_{X}\right) \rightarrow\left(\Sigma^{2}, Z_{\Sigma}\right)$ between compact connected $b$-oriented $b$-manifolds so that for each critical point $x \in \Delta$ there exist complex coordinate charts compatible with orientations induced from the $b$-orientations centered at $x$ and $f(x)$ in which $f$ takes the form $f: \mathbb{C}^{n} \rightarrow \mathbb{C}, f\left(z_{1}, \ldots, z_{n}\right)=z_{1}^{2}+\cdots+z_{n}^{2}$.

Remark 4.4. Given a $b$-Lefschetz fibration $f:\left(X, Z_{X}\right) \rightarrow\left(\Sigma, Z_{\Sigma}\right)$, the local model for $f$ around critical points $x \in \Delta$ implies ker $d f_{x}=T_{x} X$. Because $f$ is a $b$-map so is transverse to $Z_{\Sigma}$, we conclude that $Z_{\Sigma}$ and $\Delta^{\prime}$ are disjoint. The $b$-orientation induces an orientation away from the singular locus so that it makes sense to demand compatibility of the charts.

Alternatively, we could first define the notion of a $b$-achiral Lefschetz fibration and then note that its critical values must be disjoint from the singular locus, so that we can then further demand compatibility of the charts specifying the local model of $f$.

Proposition 4.5. Let $f: X^{2 n} \rightarrow \Sigma^{2}$ be an achiral Lefschetz fibration between compact connected manifolds which is injective on critical points. Assume that the generic fiber $F$ is orientable and $[F] \neq 0 \in H_{2 n-2}(X ; \mathbb{R})$. Then there exists a hypersurface $Z_{\Sigma} \subset \Sigma$ so that $f:\left(X^{2 n}, Z_{X}\right) \rightarrow\left(\Sigma^{2}, Z_{\Sigma}\right)$ is a b-Lefschetz fibration, where $Z_{X}=f^{-1}\left(Z_{\Sigma}\right)$.

Proof. We deal with orientations as in [9, Theorem 6.7]. Fix an orientation for the generic fiber $F$. As $[F] \neq 0$, this forces $f: X \backslash \Delta \rightarrow \Sigma \backslash \Delta^{\prime}$ to be an orientable fibration, which in turn orients all fibers, including the singular ones. We conclude that $X$ is orientable if and only if $\Sigma$ is. If they are, choose orientations and split $\Delta^{\prime}$ into disjoint sets $\Delta_{+}^{\prime}$ and $\Delta_{-}^{\prime}$ according to the sign of the critical points. Then, pick a separating curve $\gamma \subset \Sigma$ disjoint from $\Delta^{\prime}$ such that its interior contains all of $\Delta_{-}^{\prime}$ and no points from $\Delta_{+}^{\prime}$. If $\Sigma$ is not orientable, there instead exists a curve $\gamma \subset \Sigma$ so that $\Sigma \backslash \gamma$ is orientable, hence so is $X \backslash f^{-1}(\gamma)$. Choose orientations and then homotope $\gamma$ through negative critical values of $f$ so that all critical points are positive. Define $Z_{\Sigma}:=\gamma$ and let $Z_{X}:=f^{-1}\left(Z_{\Sigma}\right)$. Because $Z_{\Sigma}$ does not hit $\Delta^{\prime}$, it is immediate that $f$ is a $b$-map from $\left(X, Z_{X}\right)$ to $\left(Y, Z_{\Sigma}\right)$. Moreover, the orientations we chose give the appropriate $b$-orientations. But then $f$ is a $b$-Lefschetz fibration.

Remark 4.6. The curve $\gamma$ used in the previous proof is not unique. For example, in the orientable case we chose a separating curve, but we could just as well have chosen disjoint curves around each negative critical point separately. The effect of this is that the $b$-manifold structures that are used are not unique either.

Given a four-dimensional $b$-Lefschetz fibration, the $b$-version of Gompf's proof of $[19$, Theorem 10.2.18] in terms of tamed almost-complex structures supplies us with a $b$-symplectic form on $\left(X, Z_{X}\right)$, giving a log-symplectic structure on $X$.

Theorem 4.7. Let $f:\left(X^{4}, Z_{X}\right) \rightarrow\left(\Sigma^{2}, Z_{\Sigma}\right)$ be a b-Lefschetz fibration between compact connected b-oriented $b$-manifolds which is injective on critical points. Assume that the generic fiber $F$ is orientable and $[F] \neq 0 \in H_{2}(X ; \mathbb{R})$. Then $\left(X, Z_{X}\right)$ admits a log-symplectic structure.

Proof. Note that the exact sequence in homotopy, $\pi_{1}(F) \rightarrow \pi_{1}(X) \rightarrow \pi_{1}(Y) \rightarrow \pi_{0}(F) \rightarrow 0$, which was used in the proof of Theorem 3.7, is also available for Lefschetz fibrations by [19, Proposition 8.1.9] because neighbourhoods of critical values admit specific sections. This still 
holds for achiral Lefschetz fibrations and hence also for $b$-Lefschetz fibrations. If necessary, use this exact sequence in homotopy to lift $f$ to a cover of $\Sigma$ so that $f$ has connected fibers. Fix an orientation for the generic fiber $F$, which orients ker $d f$ and $\operatorname{ker}^{b} d f$ at regular points, using Proposition 2.14. As $[F] \neq 0$, this forces $f:\left(X \backslash f^{-1}\left(\Delta^{\prime}\right), Z_{X} \backslash f^{-1}\left(\Delta^{\prime}\right)\right) \rightarrow\left(Y \backslash \Delta^{\prime}, Z_{Y} \backslash \Delta^{\prime}\right)$ to be an orientable $b$-fibration. Let $c \in H^{2}(X ; \mathbb{R})$ be a class dual to $[F] \in H_{2}(X ; \mathbb{R})$, i.e. such that $\langle c,[F]\rangle=1$. Let ${ }^{b} \pi \in \Gamma\left({ }^{b} T \Sigma\right)$ be a transverse section specifying the $b$-orientation of $\left(\Sigma, Z_{\Sigma}\right)$. Then by Proposition $2.9, \rho_{\Sigma}\left({ }^{b} \pi\right)$ is a log-symplectic structure on $\left(X, Z_{\Sigma}\right)$, and $\omega_{\Sigma}:=\left({ }^{b} \pi\right)^{-1}$ is a $b$-symplectic form on $\left(\Sigma, Z_{\Sigma}\right)$.

We show the conditions of Theorem 3.4 are satisfied. Use the proof of Theorem 3.7 to obtain a $\left(\omega_{\Sigma}, f\right)$-tame $b$-almost-complex structure $J$ on $\left.{ }^{b} T X\right|_{X \backslash f^{-1}\left(\Delta^{\prime}\right)}$. As $\Delta^{\prime}$ is disjoint from $Z_{\Sigma}$, let $V \subset \Sigma$ be the disjoint union of open balls $V_{y}$ disjoint from $Z_{\Sigma}$ and centered at each point $y \in \Delta^{\prime}$. Set $W:=f^{-1}(V) \subset X$ to be the union of the neighbourhoods $W_{y}:=f^{-1}\left(V_{y}\right)$ of singular fibers $F_{y}$. Let $C \subset X$ be the disjoint union of open balls $C_{y}$ centered at each point $f^{-1}(y)=x \in \Delta_{y}$ for all $y \in \Delta^{\prime}$ disjoint from $Z_{X}$ so that on each ball $f$ takes on the local form in Definition 4.3. Possibly shrink $C$ so that $\overline{C_{y}} \subset W_{y}$. The local description of $f$ gives an almost-complex structure on $C$ with the fibers being holomorphic, and we glue this to the existing $J$ on $X \backslash C$ using a trivial version of Proposition 5.7 to obtain a global $\left(\omega_{\Sigma}, f\right)$-tame $b$-almost-complex structure $J$ on $\left(X, Z_{X}\right)$.

Let $y \in Y$ be given. If $y \in Y \backslash \Delta^{\prime}$, proceed as in the proof of Theorem 3.7 to obtain a neighbourhood $W_{y}$ of $F_{y}$ and a closed two-form $\eta_{y} \in \Omega^{2}\left(W_{y}\right)$ such that $\left[\eta_{y}\right]=\left.c\right|_{W_{y}}$ and such that $\eta_{y}$ tames $J$ on $\operatorname{ker}^{b} d f_{x}$ for all $x \in W_{y}$. If $y \in \Delta^{\prime}$, the singular fiber $F_{y}$ either is indecomposable or consists of two irreducible components $F_{y}^{ \pm}$which satisfy $\left[F_{y}^{+}\right] \cdot\left[F_{y}^{-}\right]=1$ and $\left[F_{y}^{ \pm}\right]^{2}=-1$, see [19]. In the latter case, note that $0<1=\langle c,[F]\rangle=\left\langle c,\left[F_{y}\right]\right\rangle=\left\langle c,\left[F_{y}^{+}\right]\right\rangle+$ $\left\langle c,\left[F_{y}^{-}\right]\right\rangle$. If either term is nonpositive assume without loss of generality that $\left\langle c,\left[F_{y}^{-}\right]\right\rangle=r \leq 0$. Define $c^{\prime}:=c+\left(\frac{1}{2}-r\right) c_{y}^{+}$, where $c_{y}^{+} \in H^{2}(X ; \mathbb{R})$ is a class dual to $\left[F_{y}^{+}\right]$. As $\left[F_{y}\right] \cdot\left[F_{y}^{ \pm}\right]=0$ we then have $\left\langle c^{\prime},[F]\right\rangle=\langle c,[F]\rangle>0$, and furthermore $\left\langle c^{\prime},\left[F_{y}^{+}\right]\right\rangle=\left\langle c,\left[F_{y}^{+}\right]\right\rangle+\left(\frac{1}{2}-r\right)>0$ and $\left\langle c^{\prime},\left[F_{y}^{-}\right]\right\rangle=\frac{1}{2}>0$. Moreover, as different fibers do not intersect, we have $\left.c\right|_{W_{y^{\prime}}}=\left.c^{\prime}\right|_{W_{y^{\prime}}}$ for $y^{\prime} \neq y$. After finitely many repetitions, at most once for each $y \in \Delta^{\prime}$, one obtains a class, again denoted by $c$, pairing positively with every fiber component (see [19, Exercise 10.2.19]).

Return to $y \in \Delta^{\prime}$ and let $\sigma$ be the standard symplectic form on $C_{y}$ given locally in real coordinates by $\sigma=d x_{1} \wedge d y_{1}+d x_{2} \wedge d y_{2}$, where $z_{i}=x_{i}+i y_{i}$. As all fibers $F_{y}^{\prime}$ in $C_{y}$ are holomorphic, $\left.\rho_{X}^{*} \sigma\right|_{F_{y^{\prime}} \cap C_{y}}$ tames ${ }^{b} J$ for all $y^{\prime} \in f\left(C_{y}\right)$, so that $\rho_{X}^{*} \sigma$ tames $J$ on $C_{y}$. Let $\sigma_{y}$ be an extension of $\sigma$ to $F_{y}$ as a positive area form with total area $\left\langle\sigma_{y},\left[F_{y}\right]\right\rangle$ equal to $\left\langle c,\left[F_{y}\right]\right\rangle$. Let $p: W_{y} \rightarrow F_{y}$ be a retraction and let $f: C_{y} \rightarrow[0,1]$ be a smooth radial function so that $f \equiv 0$ in a neighbourhood of $x$ and $f \equiv 1$ in a neighbourhood of $\partial C_{y}$, which is smoothly extended to $W_{y}$ by being identically 1 outside $C_{y}$. On the ball $C_{y}$, the form $\sigma$ is exact, say equal to $\sigma=d \alpha$ for $\alpha \in \Omega^{1}\left(C_{y}\right)$. Define a two-form $\eta_{y}$ on $W_{y}$ by $\eta_{y}:=p^{*}\left(f \sigma_{y}\right)+d((1-f) \alpha)$, which is closed as $f \sigma_{y}$ is a closed area form on $F_{y}$. Near $x$ we have $f \equiv 0$ so that $\eta_{y}=d \alpha=\sigma$, hence there $\rho_{X}^{*} \eta_{y}=\rho_{X}^{*} \sigma$ tames $J$, hence in particular tames $\left.J\right|_{\operatorname{ker}^{b} d f}$. Similarly, $\sigma_{y}$ is an area form on $F_{y} \backslash\{x\}$ for the orientation given by $J$, using Proposition 2.14. But then $\rho_{X}^{*} \sigma_{y}$ tames $J$ on $T F_{y}=\operatorname{ker} d f \cong \operatorname{ker}^{b} d f$ on $F_{y}$, so that the same holds for $\rho_{X}^{*} \eta_{y}$ as this condition is convex. By openness of the taming condition, shrinking $V_{y}$ and hence $W_{y}$ and possibly $C_{y}$ we can ensure that $\rho_{X}^{*} \eta_{y}$ tames $\left.J\right|_{\text {ker }^{b} d f}$ on $W_{y}$. Finally, note that $\left[\eta_{y}\right]=\left.c\right|_{W_{y}} \in H^{2}\left(W_{y} ; \mathbb{R}\right)$ by construction. We have now obtained the required neighbourhoods $W_{y}$ and forms $\eta_{y}$ for all $y \in Y$ so that by Theorem 3.4 we obtain a log-symplectic structure on $\left(X, Z_{X}\right)$. 
Proof of Theorem 4.2. This follows as an immediate corollary to Proposition 4.5 and Theorem 4.7. We require that $f$ is injective on critical points so that critical values also obtain a sign, allowing us to group them appropriately. Here we note that we can always perturb $f$ so that it is injective on critical points. If one does not want to assume this one can proceed as in [18, Lemma 3.3].

Remark 4.8. The log-symplectic structure constructed in the proof of Theorem 4.2 has connected singular locus, and may be empty. By Remark 4.6 we could have easily ensured that the singular locus has multiple components. Moreover, by the following theorem we can add components to the singular locus at will using a neighbourhood of a fiber. In particular we can always ensure the log-symplectic structure is bona fide.

Theorem 4.9 ([9, Theorem 5.1]). Let $\left(X^{2 n}, Z_{X}, \pi\right)$ be a compact log-symplectic manifold and let $k>0$ be an integer. Assume that $X$ has a compact symplectic submanifold $F^{2 n-2} \subset X \backslash Z_{X}$ with trivial normal bundle. Then $\left(X, Z_{X}^{\prime}\right)$ admits a log-symplectic structure $\pi^{\prime}$ agreeing with $\pi$ away from $Z_{X}^{\prime} \backslash Z_{X}$, where $Z_{X}^{\prime}$ is the disjoint union of $Z_{X}$ with $k$ copies of $F \times S^{1}$.

It is an interesting question whether every log-symplectic structure on a four-manifold can be obtained out of an achiral Lefschetz fibration using the construction of Theorem 4.2. This parallels the development by Gompf and Donaldson between Lefschetz pencils and symplectic structures on four-manifolds. The first thing to note is that in our construction the fibers are always contained in the eventual singular locus, or are disjoint from it. Moreover, all log-symplectic structures we construct are proper, in that all connected components of their singular loci are compact and have a compact symplectic leaf. This implies compact $\log$-symplectic four-manifolds $\left(X, Z_{X}, \pi\right)$ for which $\pi$ is not proper are not reached by our construction. Note however that one can deform $\pi$ to a proper log-symplectic structure if all components of $Z_{X}$ are compact [9, Theorem 3.6]. More serious is the fact that in our construction the singular locus fibers over a circle in the base with specified diffeomorphism type of the fiber. Hence our construction cannot create log-symplectic four-manifolds $X$ with a disconnected singular locus $Z_{X}$ with at least two components not fibering over $S^{1}$ with the same genus fiber. The following example shows this can indeed happen.

Example 4.10. Let $X^{4}=\Sigma_{g} \times \Sigma_{h}$ be the product of compact surfaces of genera $g \geq 2$ and $h \geq 1$ carrying the product symplectic form, and consider the map $f: X \rightarrow \Sigma_{h}$ given by projection. Consider a copy of the torus $T^{2}=S^{1} \times S^{1}$ by picking an essential circle in both the base and the fiber. It is Lagrangian and homologically nontrivial, so that by a result of Gompf [14, Lemma 1.6], by a slight perturbation of the symplectic structure in a neighbourhood of the torus we find a symplectic structure on $X$ for which $T^{2}$ is symplectic. Applying Theorem 4.9 we obtain a log-symplectic structure on $\left(X, T^{3}\right)$. Now use a fiber $\Sigma_{g}$ of $f$ disjoint from the torus and apply Theorem 4.9 once more to obtain a log-symplectic structure on $\left(X, Z_{X}\right)$, where $Z_{X}=T^{3} \sqcup \Sigma_{g} \times S^{1}$.

Note that $T^{3}$ cannot fiber over $S^{1}$ with fibers of genus other than one. Any fibration $p: T^{3} \rightarrow S^{1}$ with fiber $F$ induces a long exact sequence in homotopy groups, a part of which reads $\pi_{2}\left(S^{1}\right) \rightarrow \pi_{1}(F) \rightarrow \pi_{1}\left(T^{3}\right) \rightarrow \pi_{1}\left(S^{1}\right)$, or more concretely $0 \rightarrow \pi_{1}(F) \hookrightarrow \mathbb{Z}^{3} \rightarrow \mathbb{Z}$. This shows that $\pi_{1}(F)$ injects into the Abelian group $\mathbb{Z}^{3}$, hence must itself be Abelian and cannot have torsion. By counting its rank must be two, so that that the genus of $F$ must be one, and the fibers of $p$ are tori. Similarly, the product $Y:=\Sigma_{g} \times S^{1}$ cannot fiber over $S^{1}$ with fiber $F$ being a torus. We have $b_{1}(Y)=1+b_{1}\left(\Sigma_{g}\right)=2 g+1 \geq 5$ as $g \geq 2$. However, if $F$ were a torus, $Y$ would be a mapping torus of $T^{2}$, hence $b_{1}(Y) \leq 1+b_{1}\left(T^{2}\right)=3$ which is a 
contradiction. By the discussion preceding this example we conclude that the log-symplectic structure on $\left(X, Z_{X}\right)$ cannot be obtained from the construction in Theorem 4.2.

\section{5. $b$-HYPERFIBRATIONS}

In this section we introduce a class of fibration-like maps with two-dimensional fibers that can be made to satisfy the conditions of Theorem 3.4. We call them b-hyperfibrations, as they are the $b$-analogue of the notion of a hyperpencil with empty base locus, as introduced by Gompf in [17]. After defining them we show that a $b$-hyperfibration satisfying a condition analogous to having homologically essential fibers gives rise to a log-symplectic structure.

Let $E, F \rightarrow X$ be real vector bundles over a manifold $X$ and let $T: E \rightarrow F$ be a continuous bundle map. Call a point $x \in X$ regular if $T_{x}: E_{x} \rightarrow F_{x}$ is surjective, and critical otherwise. Let $\operatorname{Reg}(T)$ denote the spaces of regular points of $T$. Form the space $P \subset E$ by

$$
P=\overline{\bigcup_{x \in \operatorname{Reg}(T)} \operatorname{ker} T_{x}}
$$

and let $P_{x}=P \cap E_{x}$ for $x \in X$. Then $P_{x}=\operatorname{ker} T_{x}$ when $x$ is regular, and otherwise $P_{x} \subset \operatorname{ker} T_{x}$ consists of all limits of sequences of vectors at regular points which are annihilated by $T$.

Definition 5.1. A point $x \in X$ is called $T$-wrapped if $\operatorname{span}_{\mathbb{R}} P_{x}$ has real codimension at most two in $\operatorname{ker} T_{x}$.

Note that all points $x \in \operatorname{Reg}(T)$ are wrapped as then $P_{x}=\operatorname{ker} T_{x}$. In our applications $\operatorname{ker} T_{x}$ will be even-dimensional, so that the wrappedness condition is immediate unless $\operatorname{rank}(E) \geq 6$. Let $f:\left(X, Z_{X}\right) \rightarrow\left(Y, Z_{Y}\right)$ be a $b$-map between compact connected $b$-manifolds. As before, let $\Delta$ and $\Delta_{y}$ be the set of critical points of the map $f: X \rightarrow Y$ and those on the fiber $F_{y}$ for $y \in Y$ respectively. We can then apply the above definition to the continuous or in fact smooth map ${ }^{b} d f:{ }^{b} T X \rightarrow{ }^{b} T Y$, noting Proposition 2.14. Note that if $X$ is four-dimensional and $Y$ is a surface, every critical point of $f$ will be automatically ${ }^{b} d f$-wrapped if $\operatorname{ker}^{b} d f$ is always even-dimensional. This is because $\operatorname{ker}^{b} d f$ is two-dimensional at regular points and the dimension of $\operatorname{ker}^{b} d f$ cannot exceed four at singular points. With this we can give the definition of a $b$-hyperfibration.

Definition 5.2. A b-hyperfibration is a $b$-map $f:\left(X^{2 n}, Z_{X}\right) \rightarrow\left(Y^{2 n-2}, Z_{Y}\right)$ between compact connected $b$-oriented $b$-manifolds so that there exists a log-symplectic structure $\omega_{Y}$ on $\left(Y, Z_{Y}\right)$ and such that

i) each critical point $x \in \Delta$ is ${ }^{b} d f$-wrapped;

ii) for each critical point $x \in \Delta$, there exists a neighbourhood of $x$ and a $b$-almost-complex structure $J_{x}$ on the $b$-manifold $\left(W_{x}, W_{x} \cap Z_{X}\right)$ such that $J_{x}$ is $\left(\omega_{Y}, f\right)$-tame;

iii) for each $y \in Y$ there are only finitely many critical points $\Delta_{y}$ lying on its fiber $F_{y}$.

This should be compared with [17, Definition 2.4]. Note that the definition does not require $X$ nor $Y$ to be orientable. When $Z_{Y}=\emptyset$ one almost recovers the definition by Gompf of a hyperpencil with empty base locus, over an arbitrary symplectic base.

Remark 5.3. While a $b$-hyperfibration $f$ may have infinitely many critical points, note that regular points of $f$ are dense in $X$, arguing as in [17, Theorem 2.11]. If an open $W \subset X$ would consist entirely of critical points, choose a point $x_{0} \in W$ which minimizes $\operatorname{dim} \operatorname{ker}^{b} d f_{x}$, and using Proposition 2.14 note that $\operatorname{ker}^{b} d f \cong$ ker $d f$ is a smooth distribution near $x_{0}$ as it can be realized as ker $d(\pi \circ f)$ for a projection $\pi$. Then take a vector field in ker $d f$ and integrate it 
to obtain a curve of critical points all lying in a single fiber of $f$. This contradicts assumption iii) in the definition of a $b$-hyperfibration.

The wrappedness of the critical points will be used to obtain a global $\left(\omega_{Y}, f\right)$-tame $b$ almost-complex structure out of the locally existing ones. With this new notion in hand we can move on to the following result, which is the appropriate $b$-analogue of [17, Theorem 2.11]. Given a $b$-hyperfibration $f:\left(X, Z_{X}\right) \rightarrow\left(Y, Z_{Y}\right)$ and $y \in Y$ fixed, we refer to the closures of the connected components of $F_{y} \backslash \Delta_{y}$ as the components of the fiber $F_{y}$.

Theorem 5.4. Let $f:\left(X, Z_{X}\right) \rightarrow\left(Y, Z_{Y}, \omega_{Y}\right)$ be a b-hyperfibration between compact connected b-oriented b-manifolds. Assume that there exists a finite collection $S$ of sections of $f$ interescting all fiber components non-negatively and for each fiber component at least one section in $S$ intersecting positively. Then $\left(X, Z_{X}\right)$ admits a log-symplectic structure.

Note that the condition on the existence of such a collection $S$ of fibers of $f$ implies that each component of each fiber is homologically essential.

Remark 5.5. In the above theorem, note that by Proposition 2.17 sections $s \in S$ are $b$ maps $s:\left(Y, Z_{Y}\right) \rightarrow\left(X, Z_{X}\right)$ and furthermore that $\operatorname{ker}^{b} d f_{x} \oplus^{b} d s_{y}\left({ }^{b} T_{y} Y\right)={ }^{b} T_{x} X$ for all $x \in X$, where $y=f(x)$. In the proof of Theorem 5.4 we show a $b$-hyperfibration naturally gives rise to a $b$-almost-complex structure which is $\left(\omega_{Y}, f\right)$-tame so that ker ${ }^{b} d f$ carries a $b$ orientation. By Proposition 2.14 for smooth points $x \in X \backslash \Delta$ we then have an orientation for $T_{x} F_{y}=\operatorname{ker} d f_{x} \cong \operatorname{ker}^{b} d f_{x}$. Both ${ }^{b} T_{x} X$ and ${ }^{b} T_{y} Y$ carry orientations, hence so does ${ }^{b} d s_{y}\left({ }^{b} T_{y} Y\right)$. We can then define the positive intersection of $s \in S$ with $F_{y}$ by comparing the $b$-orientations on these tangent spaces in the usual way. Note that $s$ must intersect fibers in smooth points of $f$ as it is a section.

The proof of Theorem 5.4 will be modelled on Gompf's proof of [17, Theorem 2.11]. There will be an interplay between two types of singular behavior, namely that of the $b$-manifold structure and that of the fibration itself. The relation between these has been discussed before in Proposition 2.14. Note that in the case of a $b$-hyperfibration $f:\left(X, Z_{X}\right) \rightarrow\left(Y, Z_{Y}\right)$ with $Z_{Y}=\emptyset$, we cannot apply [17, Theorem 2.11] directly. Indeed, there is no base locus, but instead a set of sections $S$. This is akin to obtaining a Lefschetz fibration out of a Lefschetz pencil by blowing up the base locus (see also Remark 1.1).

For $V$ a real finite-dimensional vector space, let $\mathcal{B}_{V} \subset \operatorname{Aut}(V)$ be the open set of linear operators on $V$ with no real eigenvalues, and $\mathcal{J}_{V} \subset \mathcal{B}_{V}$ the set of complex structures on $V$ in either orientation. The following lemma is proven in [17] as Corollary 4.2, loc. cit.

Lemma 5.6. Let $V, W$ be real finite-dimensional vector spaces. Then there exists a canonical real-analytic retraction $j: \mathcal{B}_{V} \rightarrow \mathcal{J}_{V}$, satisfying for all linear maps $T: V \rightarrow W$ such that $T A=B T$, that $T j(A)=j(B) T$ (whenever both sides are defined).

Because the retraction in the previous lemma is canonical we can apply it pointwise to a continuously varying map, to again obtain a continuous map. Let $E^{2 n}, F^{2 n-2} \rightarrow X$ be real oriented vector bundles over a compact manifold $X$. In what follows, a two-form on a vector bundle is a continuously varying choice of skew-symmetric bilinear form on each fiber. The next proposition can be extracted from [17, Lemma 3.2]. We include a proof for completeness.

Proposition 5.7. Let $T: E \rightarrow F$ be a continuous bundle map and $\omega_{F}$ a non-degenerate two-form on $F$. Assume that for all $x \in X$ there exists a neighbourhood $W_{x}$ of $x$ with an $\left(\omega_{F}, T\right)$-tame complex structure on $\left.E\right|_{W_{x}}$. Assume that each critical point $x \in \operatorname{Crit}(T)$ is wrapped. Then there exists a continuous $\left(\omega_{F}, T\right)$-tame complex structure $J$ on E. 
Proof. Cover $X$ by open sets $W_{\alpha}$ equipped with complex structures $J_{\alpha}$ on $\left.E\right|_{W_{\alpha}}$ which are $\left(\omega_{F}, T\right)$-tame. Let $\left\{\varphi_{\alpha}\right\}$ be a subordinate partition of unity, and define

$$
A:=\sum_{\alpha} \varphi_{\alpha} J_{\alpha}: E \rightarrow E, \quad B:=\sum_{\alpha} \varphi_{\alpha} T_{*} J_{\alpha}: T(E) \rightarrow T(E),
$$

so that $T A=B T$. Since $\operatorname{ker} T$ is $J_{\alpha}$-complex for all $\alpha$ because $J_{\alpha}$ is $\left(\omega_{F}, T\right)$-tame (see below Definition 3.1), $J_{\alpha}$ descends to a map $T_{*} J_{\alpha}: T(E) \rightarrow T(E)$, hence $B$ is well-defined. In order to apply Lemma 5.6 we show that $A_{x} \in \mathcal{B}_{E_{x}}$ for all $x \in X$, i.e. that $A$ has no real eigenvalues. Let $\lambda$ be an eigenvalue of $A$ with eigenvector $v \in E$. Then $B T v=T A v=T \lambda v=\lambda T v$, so either $T v=0$, or $T v$ is a $\lambda$-eigenvector for $B$. As each $T_{*} J_{\alpha}$ is $\omega_{F}$-tame, $B$ has no real eigenvalues on any fiber. Indeed, for $0 \neq w \in T(E)$, we have $\omega_{F}(w, w)=0$. Hence if $B w=\lambda w$ for some $\lambda \in \mathbb{R}$, we have

$$
0=\omega_{F}(w, \lambda w)=\omega_{F}(w, B w)=\omega_{F}\left(w, \sum_{\alpha} \varphi_{\alpha} T_{*} J_{\alpha} w\right)=\sum_{\alpha} \varphi_{\alpha} \omega_{F}\left(w, T_{*} J_{\alpha} w\right)>0,
$$

which is a contradiction. We conclude that any real eigenvector of $A$ must lie in $\operatorname{ker} T$. Let $x \in X$ be given. As $T$-regular points are always $T$-wrapped, and by hypothesis the same holds for all $T$-critical points, we know that $x$ is $T$-wrapped. Recall the subspace $P \subset \operatorname{ker} T \subset E$ used in Definition 5.1. We construct a decomposition $\operatorname{span}_{\mathbb{R}} P_{x}=\bigoplus_{j} \Pi_{j}$, with each $\Pi_{j}$ a real two-plane which is a $J_{\alpha}$-complex line for all $J_{\alpha}$ defined on $E_{x}$. Let $v \in P_{x}$ be given. By definition of $P$, there exists a sequence $\left(x_{i}\right)_{i \in \mathbb{N}}$ of $T$-regular points converging to $x$ and elements $v_{i} \in \operatorname{ker} T_{x_{i}}$ such that $v=\lim _{i \rightarrow \infty} v_{i}$. As the points $x_{i}$ are $T$-regular, the subspaces $\operatorname{ker} T_{x_{i}}$ are two-planes in $E_{x_{i}}$ oriented by the fiber-first convention. Pass to a subsequence so that the ker $T_{x_{i}}$ converge to an oriented two-plane $\Pi \subset P_{x}$ containing $v$. Consider an open $W_{\alpha}$ containing $x$. Then there exists an $N_{\alpha} \in \mathbb{N}$ such that $x_{i} \in W_{\alpha}$ for all $i \geq N_{\alpha}$. But then for all $i \geq N_{\alpha}, \operatorname{ker} T_{x_{i}}$ is a $J_{\alpha}$-complex line, hence so is their limit $\Pi$. We conclude that $\Pi$ is a $J_{\alpha}$-complex line for each $J_{\alpha}$ defined at $x$. Proceed by induction to constructed multiple such real oriented two-planes $\Pi_{j} \subset P_{x}$ so that $\operatorname{span}_{\mathbb{R}} P_{x}=\bigoplus_{j} \Pi_{j}$, with each $\Pi_{j}$ being a $J_{\alpha}$-complex line for all $J_{\alpha}$ defined at $x$. Consider the quotient $Q_{x}:=\operatorname{ker} T_{x} / \operatorname{span}_{\mathbb{R}} P_{x}$, which inherits an orientation from $\operatorname{ker} T_{x}$, which in turn is oriented as it is $J_{\alpha}$-complex for all $\alpha$ defined at $x$, all of which are $\left(\omega_{F}, T\right)$-tame. Then $Q_{x}$ inherits complex structures $\bar{J}_{\alpha}$ from each $J_{\alpha}$ defined at $x$, and these are all compatible with the orientation on $Q_{x}$. As $x$ is $T$-wrapped, $\operatorname{dim}_{\mathbb{C}} Q_{x} \leq 1$. But then there exists a fixed nondegenerate skew-symmetric bilinear form $\omega_{x}$ on $Q_{x}$ so that all $\bar{J}_{\alpha}$ are $\omega_{x}$-tame, as one can just pick an $\omega_{x}$ giving the orientation on $Q_{x}$. Consider the $\operatorname{map} \bar{A}_{x}:=\sum_{\alpha} \varphi_{\alpha}(x) \bar{J}_{\alpha}: Q_{x} \rightarrow Q_{x}$. Then $\bar{A}_{x}$ has no real eigenvalues on $Q_{x}$. As before, if $\bar{A}_{x} \bar{v}=\lambda \bar{v}$ for $0 \neq \bar{v} \in Q_{x}$ with $\lambda$ real, we would have

$$
0=\omega_{x}(\bar{v}, \lambda \bar{v})=\omega_{x}\left(\bar{v}, \bar{A}_{x} \bar{v}\right)=\omega_{x}\left(\bar{v}, \sum_{\alpha} \varphi_{\alpha}(x) \bar{J}_{\alpha} \bar{v}\right)=\sum_{\alpha} \varphi_{\alpha}(x) \omega_{x}\left(\bar{v}, \bar{J}_{\alpha} \bar{v}\right)>0 .
$$

As ker $T_{x} \cong \operatorname{span}_{\mathbb{R}} P_{x} \oplus Q_{x}$, we conclude that any real eigenvector of $A_{x}$ must lie in $\operatorname{span}_{\mathbb{R}} P_{x}=$ $\bigoplus_{j} \Pi_{j}$. Construct a direct sum two-form $\widetilde{\omega}_{x}=\bigoplus_{j} \omega_{j}$ on $\operatorname{span}_{\mathbb{R}} P_{x}$ which tames each $J_{\alpha}$ at $x$. Then if $A_{x} v=\lambda v$ for $0 \neq v \in \operatorname{span}_{\mathbb{R}} P_{x}$ with $\lambda$ real and $v=\bigoplus_{j} v_{j}$ with respect to the direct sum decomposition of $\operatorname{span}_{\mathbb{R}} P_{x}$,

$$
0=\widetilde{\omega}_{x}(v, \lambda v)=\widetilde{\omega}_{x}\left(v, A_{x} v\right)=\sum_{j} \omega_{j}\left(v_{j}, A_{x} v_{j}\right)=\sum_{j, \alpha} \varphi_{\alpha}(x) \omega_{j}\left(v_{j}, J_{\alpha}(x) v_{j}\right)>0 .
$$

We conclude that $A_{x}$ has no real eigenvalues, hence nor does $A$. By Lemma 5.6 we obtain from $A$ a continuous complex structure $J=j(A)$ on $E$. As $T j(A)=j(B) T$ and convex 
combinations of $\left(\omega_{F}, T\right)$-tame endomorphisms are still tamed, the resulting almost-complex structure $J$ is $\left(\omega_{F}, T\right)$-tame.

Proof of Theorem 5.4. By Proposition 2.9 we need to show the existence of a $b$-symplectic form on $\left(X, Z_{X}\right)$. This is done by appealing to Theorem 3.4, hence it suffices to construct local closed two-forms $\eta_{y}$ around fibers so that the respective $b$-forms $\rho_{X}^{*} \eta_{y}$ tame a global $b$-almostcomplex structure $J$ on $\mathrm{ker}^{b} d f$, and such that they are all cohomologous to the restriction of one global class $c \in H^{2}(X ; \mathbb{R})$. Construct around each point $x \in X$ a neighbourhood $W_{x}$ and a $b$-almost-complex structure $J_{x}$ on $\left.{ }^{b} T X\right|_{W_{x}}$ which is $\left(\omega_{Y}, f\right)$-tame. These exist by definition around critical points of $X$ and away from critical points these are constructed as in the proof of Theorem 3.7, using that the fibers of $f$ are two-dimensional. Apply Proposition 5.7 to the situation where $E={ }^{b} T X, F=f^{* b} T Y, T={ }^{b} d f$ and $\omega_{F}=f^{*} \omega_{Y}$, to obtain a global $b$-almost-complex structure $J$ on $X$ which is $\left(\omega_{Y}, f\right)$-tame. Let $S$ be the finite set of sections in the hypothesis, and define $c$ to be the Poincaré dual of $\sum_{s \in S}[\operatorname{im}(s)] \in H_{2 n-2}(X ; \mathbb{R})$. To apply Poincaré duality in the absence of an orientation on $X$, the images of the sections must be cooriented. However, this exactly means that the fibers of $f$ must be oriented. Regular fibers obtain an orientation through $J$ and Proposition 2.14, while singular fibers are oriented in their smooth locus.

Let $y \in Y$ be given. By the definition of a hyperfibration, $\Delta_{y}$ is finite. Let $C_{y} \subset X$ be the disjoint union of closed balls centered at each point in $\Delta_{y}$. Choose a closed twoform $\sigma \in \Omega^{2}\left(C_{y}\right)$ so that $\rho_{X}^{*} \sigma$ tames $J$ on $\left.\operatorname{ker}^{b} d f\right|_{\Delta_{y}}$, noting that this is a condition at a finite set of points hence can easily be satisfied. Then, as taming $J$ on $\operatorname{ker}^{b} d f$ is an open condition by Remark 3.2, after possibly shrinking the balls in $C_{y}$ we can assume that $\left.J\right|_{\operatorname{ker}^{b} d f}$ is $\rho_{X}^{*} \sigma$-tame on the entirety of $C_{y}$. As by assumption $J$ is $\left(\omega_{Y}, f\right)$-tame, $F_{y} \backslash \Delta_{y}$ is a smooth noncompact $J$-holomorphic curve in $X \backslash \Delta_{y}$, whose complex $b$-orientation from $J$ agrees with its preimage $b$-orientation. By assumption there exists for each component of $F_{y} \backslash \Delta_{y}$ a section in $S$ intersecting that component positively. Choose $C_{y}$ so that $\partial C_{y}$ is transverse to $F_{y}$ and consider the intersecting circles $F_{y} \cap \partial C_{y}$. Connect each such circle to these points of intersection by a path in $F_{y} \backslash \Delta_{y}$. Let $C_{y}^{0}$ be the disjoint union of smaller concentric closed balls around $\Delta_{y}$ disjoint from these paths and with $\partial C_{y}^{0}$ transverse to $F_{y}$. Then each component $F_{i}$ of the compact surface $F_{y} \backslash \operatorname{int}\left(C_{y}^{0}\right)$ either lies fully inside $\operatorname{int}\left(C_{y}\right)$, or has a point of intersection with a section in $S$. In the latter case we will say that $F_{i}$ intersects $S$. See the following figures for illustration.
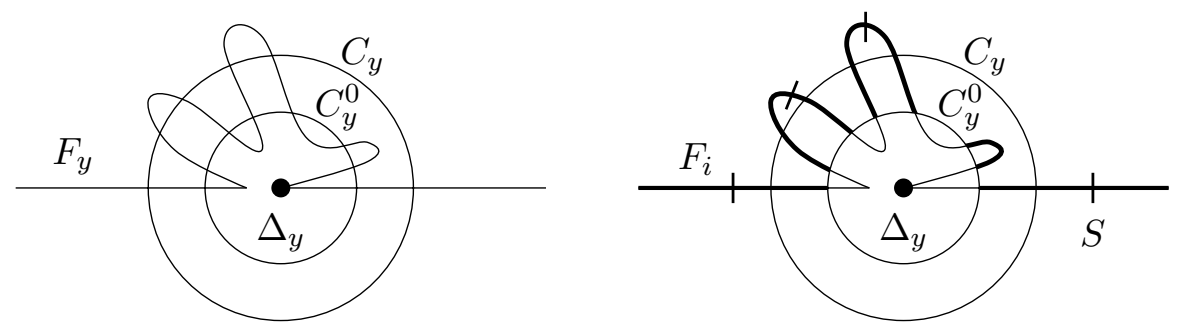

Let $W_{y}$ be the union of $\operatorname{int}\left(C_{y}^{0}\right)$ with a tubular neighbourhood rel boundary of $F_{y} \backslash \operatorname{int}\left(C_{y}^{0}\right)$ inside $X \backslash \operatorname{int}\left(C_{y}^{0}\right)$. Extend each $F_{i}$ to a closed oriented smooth surface $\widehat{F}_{i} \subset W_{y}$ by arbitrarily attaching a surface inside $C_{y}^{0}$. Then the classes $\left[\widehat{F}_{i}\right] \in H_{2}\left(W_{y} ; \mathbb{Z}\right)$ form a basis for the homology of $W_{y}$. Indeed, contracting the whole neighbourhood $C_{y}^{0}$ to $\Delta_{y}$ we see that $F_{y}$ becomes homotopy equivalent to a wedge of the $F_{i}$. This $W_{y}$ is the desired neighbourhood of $F_{y}$ on 
which we construct the form $\eta_{y}$ in the hypothesis of Theorem 3.4, using ideas similar to the proof of Theorem 4.7. Since $F_{y}$ is $J$-holomorphic with $\left.J\right|_{\operatorname{ker}^{b} d f}$ being $\rho_{X}^{*} \sigma$-tame on $C_{y},\left.\sigma\right|_{F_{i} \cap C_{y}}$ is a positive area form for each $i$. For each $F_{i}$ intersecting $S$, let $\sigma_{i}$ be an extension of $\sigma$ to $F_{i}$ as a positive area form with total area $\left\langle\sigma_{i},\left[\widehat{F}_{i}\right]\right\rangle$ equal to $\#\left(F_{i} \cap \operatorname{im}(S)\right)>0$. Let $p$ denote the tubular neighbourhood projection onto $F_{y} \backslash \operatorname{int}\left(C_{y}^{0}\right)$ and let $f: C_{y} \rightarrow[0,1]$ be a smooth radial function defined on each ball around points in $\Delta_{y}$ so that $f \equiv 0$ in a neighbourhood of $C_{y}^{0}$ and $f \equiv 1$ in a neighbourhood of $\partial C_{y}$, which is smoothly extended to $W_{y}$ by being identically 1 outside of $C_{y}$. See the following figure for illustration.

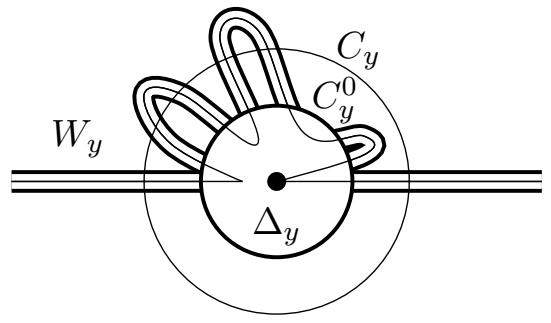

On the balls $C_{y}$, the form $\sigma$ is exact, say equal to $\sigma=d \alpha$ for $\alpha \in \Omega^{1}\left(C_{y}\right)$. Define a two-form $\eta_{y}$ on $W_{y}$ by $\eta_{y}:=\sum_{i} p^{*}\left(f \sigma_{i}\right)+d((1-f) \alpha)$. In other words, $\sigma$ is extended by 0 outside $C_{y}$, while the $\sigma_{i}$ are extended by 0 inside $C_{y}^{0}$. For all functions $f \neq 0$ the form $f \sigma_{i}$ is a closed area form defined on the surface $F_{i}$, so that $\eta_{y}$ is nonnegative and closed. On $W_{y} \cap C_{y}^{0}=C_{y}^{0}$ we have $f \equiv 0$ so that $\eta_{y}=d \alpha=\sigma$, hence there $\rho_{X}^{*} \eta_{y}=\rho_{X}^{*} \sigma$ tames $\left.J\right|_{\text {ker }^{b} d f}$. Similarly, on $F_{y} \backslash \Delta_{y}$ the forms $\sigma_{i}$ are area forms for the orientation given by $J$. But then $\rho_{X}^{*} \eta_{y}$ tames $J$ on $T_{x} F_{y}=\operatorname{ker} d f_{x} \cong \operatorname{ker}^{b} d f_{x}$ for all $x \in F_{y} \backslash \Delta_{y}$ as this condition is convex. By openness of the taming condition, narrowing the tubular neighbourhood $p$ defining $W_{y}$ ensures that $\rho_{X}^{*} \eta_{y}$ tames $\left.J\right|_{\text {ker }^{b} d f_{x}}$ for all $x \in W_{y} \backslash \operatorname{int}\left(C_{y}^{0}\right)$. But then $\rho_{X}^{*} \eta_{y}$ tames $J$ on $\operatorname{ker}^{b} d f_{x}$ for all $x \in W_{y}$.

What remains is to show that $\left.c\right|_{W_{y}}=\left[\eta_{y}\right] \in H^{2}\left(W_{y} ; \mathbb{R}\right)$. Recall that every component $F_{i}$ of $F_{y} \backslash \operatorname{int}\left(C_{y}^{0}\right)$ either intersects $S$ or lies in $\operatorname{int}\left(C_{y}\right)$. For those $F_{i}$ intersecting $S$ we have $\left\langle\left[\eta_{y}\right],\left[\widehat{F}_{i}\right]\right\rangle=\left\langle\left[\sigma_{i}\right],\left[\widehat{F}_{i}\right]\right\rangle=\#\left(F_{i} \cap \operatorname{im}(S)\right)=\left\langle c,\left[\widehat{F}_{i}\right]\right\rangle$. For $F_{i}$ disjoint from $S$ we know that $F_{i} \subset C_{y}$, but $\eta_{y}$ is exact in $C_{y}$, so that we have $\left\langle\left[\eta_{y}\right],\left[\widehat{F}_{i}\right]\right\rangle=\left\langle 0,\left[\widehat{F}_{i}\right]\right\rangle=0=\left\langle c,\left[\widehat{F}_{i}\right]\right\rangle$. As $\left[\eta_{y}\right]$ agrees with the class $\left.c\right|_{W_{y}} \in H^{2}\left(W_{y} ; \mathbb{R}\right)$ when evaluated on the classes $\left[\widehat{F}_{i}\right]$, which form a basis of $H_{2}\left(W_{y} ; \mathbb{Z}\right)$, we see that $\left[\eta_{y}\right]=\left.c\right|_{W_{y}} \in H^{2}\left(W_{y} ; \mathbb{R}\right)$ as desired. Applying Theorem 3.4 we conclude that $\left(X, Z_{X}\right)$ admits a log-symplectic structure.

\section{LOG-SYMPLECTIC AND FOLDED-SYMPLECTIC STRUCTURES}

In this section we discuss folded-symplectic structures and show that log-symplectic structures naturally give rise to folded-symplectic structures. Folded-symplectic structures are studied amongst others in $[3,6,7,8]$.

Definition 6.1. A folded-symplectic structure on a compact $2 n$-dimensional manifold $X$ is a closed two-form $\omega$ such that $\bigwedge^{n} \omega$ is transverse to the zero section in $\bigwedge^{2 n} T^{*} X$, and such that $\left.\omega^{n-1}\right|_{Z_{\omega}} \neq 0$, where $Z_{\omega}=\left(\bigwedge^{n} \omega\right)^{-1}(0)$. The hypersurface $Z_{\omega}$ is called the folding locus of $\omega$, while its complement $X \backslash Z_{\omega}$ is called the symplectic locus. A folded-symplectic structure is called bona fide if $Z_{\omega} \neq \emptyset$.

This definition should be compared with Definition 2.2. We will say the pair $\left(X, Z_{X}\right)$ admits a folded-symplectic structure if $X$ admits a folded-symplectic structure $\omega$ for which 
$Z_{\omega}=Z_{X}$. According to the Darboux model, a folded-symplectic structure $\omega$ is locally given by $\omega=x_{1} d x_{1} \wedge d x_{2}+\cdots+d x_{2 n-1} \wedge d x_{2 n}$, using coordinates $x_{i}$ in a neighbourhood $U$ such that $Z_{\omega} \cap U=\left\{x_{1}=0\right\}$.

Cannas da Silva gave a homotopical characterization for an orientable manifold to admit a folded-symplectic structure.

Theorem $6.2([7])$. Let $X$ be an orientable manifold. Then $X$ admits a folded-symplectic structure if and only if $X$ admits a stable almost-complex structure. In particular, every orientable four-manifold admits a folded-symplectic structure.

Further, Baykur has given a construction showing there is a relation between achiral Lefschetz fibrations on four-manifolds and folded-symplectic structures. This should be compared with Theorem 4.2 .

Theorem 6.3 ([3, Proposition 3.2]). Let $f: X^{4} \rightarrow \Sigma^{2}$ be an achiral Lefschetz fibration between compact connected manifolds. Assume that the generic fiber $F$ is orientable and $[F] \neq 0 \in H_{2}(X ; \mathbb{R})$. Then $X$ admits a folded-symplectic structure.

From our point of view this theorem does not come as a surprise. Indeed, every logsymplectic structure gives rise to a folded-symplectic structure, as is known in the Poisson community. We learned the proof of this result from Mărcut and Frejlich.

Theorem 6.4. Let $\left(X^{2 n}, Z_{X}, \pi\right)$ be a compact log-symplectic manifold. Then $\left(X, Z_{X}\right)$ admits a folded-symplectic structure $\omega$ for which $\omega=\pi^{-1}$ outside a neighbourhood of $Z_{X}$.

Proof. By Proposition 2.11 a neighbourhood of each connected component $Z$ of $Z_{X}$ is equivalent to a neighbourhood $U$ of the zero section of the normal bundle $N Z$ equipped with a distance function $|x|$, so that $\pi^{-1}=d \log |x| \wedge \theta+\sigma$ for closed one- and two-forms $\theta$ and $\sigma$ on $Z$ satisfying $\theta \wedge \sigma^{n-1} \neq 0$. By rescaling $|x|$ we can assume that $U$ contains all points of distance at most $e^{2}+1$ away from the zero section. Denote $\omega_{Z}=d|x|^{2} \wedge \theta+\sigma$ and let $f: \mathbb{R}_{+} \rightarrow \mathbb{R}$ be a smooth monotone interpolation between the functions $f_{0}:[0,1] \rightarrow \mathbb{R}, f_{0}(x)=x^{2}$ and $f_{1}:\left[e^{2}, \infty\right) \rightarrow \mathbb{R}, f_{1}(x)=\log x$. Consider the closed two-form $\omega_{f}=d f(|x|) \wedge \theta+\sigma$, extended by $\pi^{-1}$ outside of $U$. Then $\omega_{f}=\pi^{-1}$ away from $Z$, while near $Z$ we have $\omega_{f}=\omega_{Z}$. Moreover, $\omega_{f}$ is symplectic on $X \backslash Z$ by monotonicity of $f$. Perform this procedure for all connected components of $Z_{X}$ to obtain a closed two-form $\omega$ on $X$ for which $\omega=\pi^{-1}$ away from $Z_{X}$. By the local description near $Z_{X}$ it follows that $\omega^{n}$ vanishes transversally with $Z_{\omega}=Z_{X}$. Further, the restriction of $\omega^{n-1}$ to $Z_{\omega}$ is equal to $\sigma^{n-1}$, hence is nonvanishing as $\theta \wedge \sigma^{n-1} \neq 0$. We conclude that $\omega$ is a folded-symplectic structure for $\left(X, Z_{X}\right)$.

The previous theorem, together with Theorem 4.2, implies Theorem 6.3. Moreover, the folded-symplectic structure of Theorem 6.3 agrees with the one obtained through our methods, as is hinted at by the fact that in Baykur's construction the folding locus fibers over the circle.

The converse to Theorem 6.4 does not hold. For example, $S^{4}$ does not admit a logsymplectic structure by Theorem 2.12, while it does admit folded-symplectic structures by Theorem 6.2. Similarly, by Theorem 2.13 and results from Seiberg-Witten theory due to Taubes [30], the four-manifold $\mathbb{C} P^{2} \# \mathbb{C} P^{2}$ does not admit log-symplectic structures, bona fide or not. However, by Theorem 6.2 it does admit a folded-symplectic structure.

The reason the converse to Theorem 6.4 is false is essentially because the information contained in the one-form $\theta$ determined by the log-symplectic structure (see Proposition 2.11) 
is lost when passing to the folded-symplectic world. Note here that a folded-symplectic structure $\omega$ restricts to $Z_{\omega}$ to define a one-dimensional foliation $\operatorname{ker}\left(\left.\omega\right|_{Z_{\omega}}\right)$ called the null foliation, and $\left.\omega\right|_{Z_{\omega}}$ is a pre-symplectic structure on $Z_{\omega}$, i.e. a closed two-form of maximal rank. On the other hand, a log-symplectic structure $\pi$ induces a cosymplectic structure on $Z_{\pi}$ by Proposition 2.11, so that the associated nowhere-vanishing closed one-form gives a codimension-one foliation on $Z_{\pi}$. Further, there is a symplectic structure on the leaves.

The following result makes precise that it is exactly the existence of a suitable closed one-form $\theta$ on $Z_{X}$ that makes the converse to Theorem 6.4 hold.

Theorem 6.5. Let $\left(X^{2 n}, Z_{X}, \omega\right)$ be a compact folded-symplectic manifold. Assume that there exists a closed one-form $\theta \in \Omega^{1}\left(Z_{X}\right)$ such that $\left.\theta \wedge \omega^{n-1}\right|_{Z_{X}} \neq 0$. Then $\left(X, Z_{X}\right)$ admits a log-symplectic structure $\pi$ for which $\pi=\omega^{-1}$ outside a neighbourhood of $Z_{X}$.

In other words, when the folded-symplectic form $\omega$ can be complemented to give a cosymplectic structure on $Z_{X}$, one can turn $\omega$ into a log-symplectic structure.

Proof. Consider the normal bundle $N Z_{X}$ and let $U$ be a neighbourhood of the zero section. Choose a distance function $|x|$ for $Z_{X}$ which is constant outside of $\bar{U}$. Note that $d \log |x| \wedge \theta \wedge$ $\omega^{n-1}$ is nonzero at $Z_{X}$ as $\left.\theta \wedge \omega^{n-1}\right|_{Z_{X}} \neq 0$ and $|x|$ is transverse to $Z_{X}$. By continuity it is still nonzero in a neighbourhood $V \subset U$ of $Z_{X}$. Let $f=f(|x|)$ be a smooth function on $N Z_{X}$ so that $f \equiv 1$ on $V$ and $f \equiv 0$ near $\partial U$, which is then extended to $X$ by being identically 0 outside $U$. Define a closed $b$-two-form $\omega_{f}=t d(f \log |x|) \wedge \theta+\omega \in{ }^{b} \Omega^{2}(X)$ for the $b$-manifold $\left(X, Z_{X}\right)$, where $t \neq 0$ is a real parameter. Choose the sign of $t$ so that the forms $t d(f \log |x|) \wedge \theta$ and $\omega$ give the same orientation on $V \backslash Z_{X}$. We have $\omega_{f}^{n}=\operatorname{tn} d(f \log |x|) \wedge \theta \wedge \omega^{n-1}+\omega^{n}$, so by choosing $t$ small enough we conclude that $\omega_{f}$ is a $b$-symplectic form for which $\omega_{f}=\omega$ outside $\bar{U}$. By Proposition 2.9 the dual bivector $\pi$ to $\omega_{f}$ is a log-symplectic structure for $\left(X, Z_{X}\right)$, and $\pi=\omega^{-1}$ away from $Z_{X}$.

\section{REFERENCES}

[1] S. Akbulut and Ç. Karakurt, Every 4-manifold is BLF, J. Gökova Geom. Topol. GGT 2 (2008), 83-106.

[2] D. Auroux, S. K. Donaldson, and L. Katzarkov, Singular Lefschetz pencils, Geom. Topol. 9 (2005), 10431114.

[3] R. İ. Baykur, Kähler decomposition of 4-manifolds, Algebr. Geom. Topol. 6 (2006), 1239-1265.

[4] R. İ. Baykur, Existence of broken Lefschetz fibrations, Int. Math. Res. Not. IMRN (2008), Art. ID rnn 101,15 .

[5] R. İ. Baykur, Topology of broken Lefschetz fibrations and near-symplectic four-manifolds, Pacific J. Math. 240 (2009), no. 2, 201-230.

[6] A. Cannas da Silva, V. Guillemin, and A. R. Pires, Symplectic origami, Int. Math. Res. Not. IMRN (2011), no. 18, 4252-4293.

[7] A. Cannas da Silva, Fold-forms for four-folds, J. Symplectic Geom. 8 (2010), no. 2, 189-203.

[8] A. Cannas da Silva, V. Guillemin, and C. Woodward, On the unfolding of folded symplectic structures, Math. Res. Lett. 7 (2000), no. 1, 35-53.

[9] G. R. Cavalcanti, Examples and counter-examples of log-symplectic manifolds, arXiv:1303.6420.

[10] S. K. Donaldson, Lefschetz pencils on symplectic manifolds, J. Differential Geom. 53 (1999), no. 2, 205236.

[11] J. B. Etnyre and T. Fuller, Realizing 4-manifolds as achiral Lefschetz fibrations, Int. Math. Res. Not. (2006), Art. ID 70272, 21.

[12] P. Frejlich, D. Martínez Torres, and E. Miranda, A note on symplectic topology of b-manifolds, arXiv: 1312.7329.

[13] D. T. Gay and R. Kirby, Constructing Lefschetz-type fibrations on four-manifolds, Geom. Topol. 11 (2007), 2075-2115. 
[14] R. E. Gompf, A new construction of symplectic manifolds, Ann. of Math. (2) 142 (1995), no. 3, 527-595.

[15] R. E. Gompf, The topology of symplectic manifolds, Turkish J. Math. 25 (2001), no. 1, 43-59.

[16] R. E. Gompf, Symplectic structures from Lefschetz pencils in high dimensions, Proceedings of the Casson Fest, Geom. Topol. Monogr., vol. 7, Geom. Topol. Publ., Coventry, 2004, pp. 267-290 (electronic).

[17] R. E. Gompf, Toward a topological characterization of symplectic manifolds, J. Symplectic Geom. 2 (2004), no. 2, 177-206.

[18] R. E. Gompf, Locally holomorphic maps yield symplectic structures, Comm. Anal. Geom. 13 (2005), no. 3, $511-525$.

[19] R. E. Gompf and A. I. Stipsicz, 4-manifolds and Kirby calculus, Graduate Studies in Mathematics, vol. 20, American Mathematical Society, Providence, RI, 1999.

[20] V. Guillemin, E. Miranda, and A. R. Pires, Symplectic and Poisson geometry on b-manifolds, Adv. Math. 264 (2014), 864-896.

[21] K. Honda, Local properties of self-dual harmonic 2-forms on a 4-manifold, J. Reine Angew. Math. 577 (2004), 105-116.

[22] R. L. Klaasse, Ph.D. thesis, Utrecht University, 2016. Work in progress.

[23] C. LeBrun, Yamabe constants and the perturbed Seiberg-Witten equations, Comm. Anal. Geom. 5 (1997), no. $3,535-553$.

[24] Y. Lekili, Wrinkled fibrations on near-symplectic manifolds, Geom. Topol. 13 (2009), no. 1, 277-318. Appendix B by R. İnanç Baykur.

[25] I. Mărcut and B. Osorno Torres, Deformations of log-symplectic structures, J. Lond. Math. Soc. (2) 90 (2014), no. 1, 197-212.

[26] I. Mărcut and B. Osorno Torres, On cohomological obstructions for the existence of log-symplectic structures, J. Symplectic Geom. 12 (2014), no. 4, 863-866.

[27] D. McDuff and D. Salamon, Introduction to symplectic topology, second ed., Oxford Mathematical Monographs, The Clarendon Press, Oxford University Press, New York, 1998.

[28] R. B. Melrose, The Atiyah-Patodi-Singer index theorem, Research Notes in Mathematics, vol. 4, A K Peters, Ltd., Wellesley, MA, 1993.

[29] O. Radko, A classification of topologically stable Poisson structures on a compact oriented surface, J. Symplectic Geom. 1 (2002), no. 3, 523-542.

[30] C. H. Taubes, The Seiberg-Witten invariants and symplectic forms, Math. Res. Lett. 1 (1994), no. 6, 809-822.

[31] W. P. Thurston, Some simple examples of symplectic manifolds, Proc. Amer. Math. Soc. 55 (1976), no. 2, $467-468$.

Department of Mathematics, Utrecht University, 3508 TA Utrecht, The Netherlands

E-mail address: g.r.cavalcanti@uu.nl

Department of Mathematics, Utrecht University, 3508 TA Utrecht, The Netherlands

E-mail address: r.l.klaasse@uu.nl 